\title{
Hydroinformatic environment for coastal waters hydrodynamics and water quality modelling
}

\author{
J.L.S. Pinho ${ }^{\mathrm{a}}$, J.M. Pereira Vieira ${ }^{\mathrm{a}}$, J.S. Antunes do Carmo ${ }^{\mathrm{b}, *}$ \\ ${ }^{a}$ Department of Civil Engineering, University of Minho, Largo do Paço, 4700-320 Braga, Portugal \\ ${ }^{\mathrm{b}}$ Department of Civil Engineering, University of Coimbra, Pólo II-Pinhal de Marrocos, 3030-290 Coimbra, Portugal
}

Received 6 January 2003; accepted 30 January 2004

\begin{abstract}
A hydroinformatic environment was created with the general objective of solving environmental problems in coastal waters. This environment consists of three components: a component for data organization and treatment; a component for modelling and simulating water quality and hydrodynamics; and a component for analysing, visualizing and editing the results. This paper describes the hydroinformatic components and the major developments introduced: different methodologies for analysing the performance of finite elements meshes, a conditioned mesh refinement procedure, a three-dimensional (3D) hydrodynamic model based on the Princeton Ocean Model with an alternative finite elements technique for the external mode computation, 2D and 3D water quality models for coastal waters and a methodology for GIS model results integration. The modular approach adopted in the development of this hydroinformatic environment appears to be a very suitable and versatile methodology for decision support systems to be applied in coastal zones environment management.
\end{abstract}

(C) 2004 Elsevier Ltd. All rights reserved.

Keywords: Hydroinformatics; Mathematical modelling; Hydrodynamics; Water quality; Coastal waters

\section{Introduction}

Coastal zones are very attractive regions for human settlement, but anthropogenic activities may have significant environmental impacts on these sensitive natural systems. The approach to water management is dictated by public safety, economic and environmental considerations. Hydroinformatics is a new scientific branch linking informatics tools with hydraulics and environmental concepts and models, providing both operational information and insights into long-term trends [1]. The rapidly growing possibilities of computational resources, as well as the user friendly processing of spatial information and graphical presentation developments, has the potential to provide novel and improved tools to support the planning and management of coastal zones.

A major effort has been made over the last decade to integrate hydrodynamic and water quality models for coastal water studies. In these natural systems, the use of

\footnotetext{
* Corresponding author. Tel.: + 351-239-797-153; fax: +351-239-797-152.

E-mail address: jsacarmo@dec.uc.pt (J.S. Antunes do Carmo).
}

mathematical models for the accurate simulation of circulation patterns and biogeochemical processes is a very powerful method that greatly enhances the decision support tools used for water resources management.

The purpose of this paper is to present a hydroinformatic environment that has been created with the overall objective of solving environmental problems in coastal waters. This environment consists of three components: a component for data organization and processing; a component for modelling and simulating water quality and hydrodynamics, and a component to analyse, visualize, and edit the results. The modular approach adopted in the development of this hydroinformatics environment appears to be a very appropriate and versatile methodology, applicable to decision support systems that may be adopted for coastal zone environment management.

\section{Hydroinformatic environment}

The hydroinformatic environment consists of the following three principal components: (1) two-dimensional (2DH) 


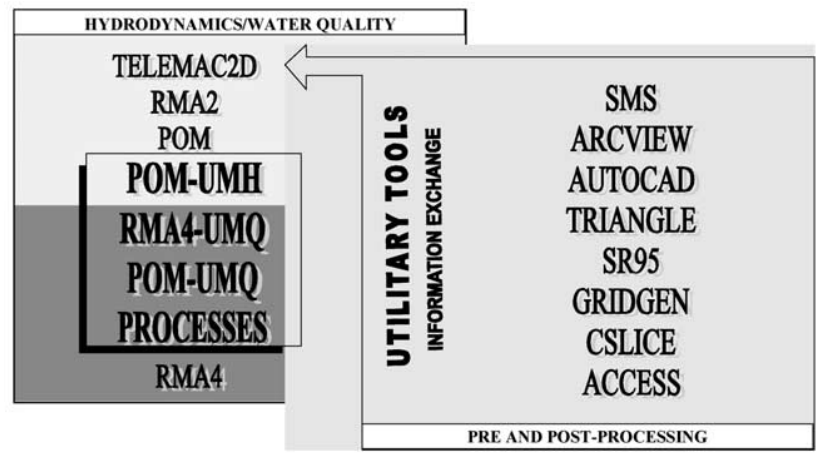

Fig. 1. Hydroinformatic environment.

and quasi-three-dimensional (quasi-3D) hydrodynamic models. These models can simulate current patterns induced by tides and wind. Moreover, with the quasi-3D hydrodynamic model, Princeton Ocean Model-UMH (POM-UMH), which was developed from the POM [2], the $3 \mathrm{D}$ features of currents driven by wind in a stratified water column can be properly simulated; (2) two water quality models that can integrated into the hydroinformatic environment in order to simulate water quality problems involving an unlimited number of constituents, and any kind of water quality processes, within both two and 3D domains [3]. The first water quality model consists of an improved version of the RMA4 program [4] and the second is an improved version of the POM program, which is able to study the advection-dispersion-reaction of an unlimited number of biogeochemical constituents; (3) pre- and postprocessing tasks were carried out with the surface modelling system (SMS) software [4], data base tools and a geographical information systems (GIS) tool [5]. Fig. 1 depicts the hydroinformatic environment built up, showing the models that have been improved or undergone innovative modifications.

Hydrodynamics modelling, is carried out utilizing the TELEMAC2D [6] and RMA2 [7] 2DH models and the quasi-3D models, POM and POM-UMH. An improvement has been introduced into POM whereby the external model is calculated using a finite element method technique in order to enhance its overall performance when applied to geometrically complex problems in which specific boundary conditions are considered.

The quasi-3D models solve the following mass and momentum conservation equations [2]:

$$
\begin{aligned}
& \frac{\partial \eta}{\partial t}+\frac{\partial}{\partial x}(u H)+\frac{\partial}{\partial y}(v H)+\frac{\partial w}{\partial \sigma}=0 \\
& \frac{\partial}{\partial t}(u H)+\frac{\partial(u u H)}{\partial x}+\frac{\partial(v u H)}{\partial y}+\frac{\partial}{\partial \sigma}(w u)-f v H \\
& =-H P_{1}+\frac{\partial}{\partial \sigma}\left(\frac{K_{\mathrm{M}}}{H} \frac{\partial u}{\partial \sigma}\right)+H F_{1}
\end{aligned}
$$

$$
\begin{gathered}
\frac{\partial}{\partial t}(v H)+\frac{\partial(u v H)}{\partial x}+\frac{\partial(v v H)}{\partial y}+\frac{\partial}{\partial \sigma}(w v)+f u H \\
=-H P_{2}+\frac{\partial}{\partial \sigma}\left(\frac{K_{\mathrm{M}}}{H} \frac{\partial v}{\partial \sigma}\right)+H F_{2}
\end{gathered}
$$

where $x$ and $y$ are the horizontal Cartesian coordinates, $\sigma$ is the sigma vertical coordinate, $t$ is the time, $u$ and $v$ are the horizontal velocity components, $w$ is the transformed vertical velocity (physically, $w$ is the velocity component normal to sigma surfaces), $H \equiv h+\eta$ is the total depth ( $h(x, y)$ is the bottom topography and $\eta(x, y, t)$ is the surface elevation), $f$ is the Coriolis parameter, $P_{1}$ and $P_{2}$ are the horizontal pressure gradients terms, $F_{1}$ and $F_{2}$ are the horizontal diffusion terms, and $K_{\mathrm{M}}$ is the vertical kinematic viscosity.

The transformation of $w$ to the cartesian vertical velocity is:

$$
\begin{aligned}
W= & w+u\left(\sigma \frac{\partial H}{\partial x}+\frac{\partial \eta}{\partial x}\right)+v\left(\sigma \frac{\partial H}{\partial y}+\frac{\partial \eta}{\partial y}\right) \\
& +\sigma \frac{\partial H}{\partial t}+\frac{\partial \eta}{\partial t}
\end{aligned}
$$

Complete thermodynamics have been implemented for the models, considering the following potential temperature $(\Theta)$ and salinity $(S)$ transport equations:

$$
\begin{aligned}
& \frac{\partial}{\partial t}(\Theta H)+\frac{\partial(u \Theta H)}{\partial x}+\frac{\partial(v \Theta H)}{\partial y}+\frac{\partial}{\partial \sigma}(w \Theta) \\
& =\frac{\partial}{\partial \sigma}\left(\frac{K_{\mathrm{H}}}{H} \frac{\partial \Theta}{\partial \sigma}\right)+H F_{\Theta} \\
& \frac{\partial}{\partial t}(S H)+\frac{\partial(u S H)}{\partial x}+\frac{\partial(v S H)}{\partial y}+\frac{\partial}{\partial \sigma}(w S) \\
& =\frac{\partial}{\partial \sigma}\left(\frac{K_{\mathrm{H}}}{H} \frac{\partial S}{\partial \sigma}\right)+H F_{\mathrm{S}}
\end{aligned}
$$

where $K_{\mathrm{H}}$ is the vertical diffusivity and $F_{\Theta}, F_{\mathrm{S}}$ are the horizontal heat and salt diffusion terms, respectively. A second moment turbulence closure sub-model [2] to provide vertical mixing coefficients, based on the transport of the scalar quantities $q^{2}$ and $q^{2} l$ is implemented:

$$
\begin{gathered}
\frac{\partial}{\partial t}\left(q^{2} H\right)+\frac{\partial\left(u q^{2} H\right)}{\partial x}+\frac{\partial\left(v q^{2} H\right)}{\partial y}+\frac{\partial}{\partial \sigma}\left(w q^{2}\right) \\
=\frac{\partial}{\partial \sigma}\left(\frac{K_{\mathrm{q}}}{H} \frac{\partial q^{2}}{\partial \sigma}\right)+\frac{2 K_{\mathrm{M}}}{H}\left[\left(\frac{\partial u}{\partial \sigma}\right)^{2}+\left(\frac{\partial v}{\partial \sigma}\right)^{2}\right] \\
+2 K_{\mathrm{H}} \frac{g}{\rho} \frac{\partial \rho}{\partial \sigma}-2 \frac{q^{3} H}{B_{1} l}+H F_{\mathrm{q}}
\end{gathered}
$$




$$
\begin{aligned}
& \frac{\partial}{\partial t}\left(q^{2} l H\right)+\frac{\partial\left(u q^{2} l H\right)}{\partial x}+\frac{\partial\left(v q^{2} l H\right)}{\partial y}+\frac{\partial}{\partial \sigma}\left(w q^{2} l\right) \\
& =\frac{\partial}{\partial \sigma}\left(\frac{K_{\mathrm{q}}}{H} \frac{\partial\left(q^{2} l\right)}{\partial \sigma}\right)+K_{\mathrm{M}} E_{1} \frac{l}{H}\left[\left(\frac{\partial u}{\partial \sigma}\right)^{2}+\left(\frac{\partial v}{\partial \sigma}\right)^{2}\right] \\
& +K_{\mathrm{H}} E_{3} l \frac{g}{\rho} \frac{\partial \rho}{\partial \sigma}-\frac{q^{3} H}{B_{1}}\left[1+E_{2}\left(\frac{l}{k L}\right)^{2}\right]+H F_{1}
\end{aligned}
$$

where $q$ is the turbulence kinetic energy, $l$ is the turbulence length scale, $g$ is the gravity, $\rho$ is the water density, $k L$ is the Von-Kármán constant, and $F_{\mathrm{q}}, F_{1}$ are horizontal diffusion terms. Constants $B_{1}, E_{1}, E_{2}$ and $E_{3}$ values are presented in Ref. [2].

2DH hydrodynamic models are based on the vertically integrated mass and momentum conservation equations:

$$
\begin{aligned}
& \frac{\partial \eta}{\partial t}+\frac{\partial[(h+\eta) U]}{\partial x}+\frac{\partial[(h+\eta) V]}{\partial y}=0 \\
& \frac{\partial U}{\partial t}+U \frac{\partial U}{\partial x}+V \frac{\partial U}{\partial y} \\
& =f V-g \frac{\partial \eta}{\partial x}-\frac{g}{\rho} \frac{\partial \rho}{\partial x} \frac{h+\eta}{2}+\frac{\rho_{\mathrm{a}} k W_{\mathrm{v}}^{2} \cos \varphi}{h+\eta} \\
& \quad-\frac{g U \sqrt{U^{2}+V^{2}}}{(h+\eta) C^{2}}+\frac{\varepsilon}{\rho}\left(\frac{\partial^{2} U}{\partial x^{2}}+\frac{\partial^{2} U}{\partial y^{2}}\right) \\
& \frac{\partial V}{\partial t}+U \frac{\partial V}{\partial x}+V \frac{\partial V}{\partial y} \\
& =-f U-g \frac{\partial \eta}{\partial y}-\frac{g}{\rho} \frac{\partial \rho}{\partial y} \frac{h+\eta}{2}+\frac{\rho_{\mathrm{a}} k W_{\mathrm{v}}^{2} \sin \varphi}{h+\eta} \\
& \quad-\frac{g V \sqrt{U^{2}+V^{2}}}{(h+\eta) C^{2}}+\frac{\varepsilon}{\rho}\left(\frac{\partial^{2} V}{\partial x^{2}}+\frac{\partial^{2} V}{\partial y^{2}}\right)
\end{aligned}
$$

where $U$ and $V$ are the vertical average of the horizontal velocity components, $\rho_{\mathrm{a}}$ is the air density, $W_{\mathrm{v}}$ is the wind velocity, $\varphi$ is the wind direction, $C$ is the Chezy coefficient and $\varepsilon$ is the turbulent viscosity coefficient.

Water quality modelling is a very hard task due to difficulties resulting from the complexity of the surface water ecosystems processes. Characterization and quantification of the relations between components of a specific ecosystem require a rigorous selection of the mathematical formulations to ensure that the water quality variables selected as indicators of the water quality conditions are accurately modelled. A specific model (PROCESSES) for water quality processes was developed. This model is based on a Runge-Kutta numerical integration scheme for solving the linear partial differential equation systems that usually result from the establishment of the water quality variables' mass conservation. These equations read:

$$
\frac{\partial B_{i}}{\partial t}=f\left(t, B_{1}, B_{2}, \ldots, B_{n}\right) \quad i=1, n
$$

where $B_{i}$ is the $i$ th water quality variable and $n$ is the number of equations. Several water quality processes were implemented for the most frequent water quality problems: conservative and non-conservative constituents, dissolved oxygen, and primary production in coastal waters. This model can either run in a stand-alone mode or be integrated with hydrodynamic and water quality models.

In the hydroinformatic environment implemented, water quality problems can be simulated by resorting to $2 \mathrm{DH}$ models (RMA4 and RMA4-UMQ) and a quasi-3D model (POMUMQ). For this last model an additional transport equation is added to Eqs. (1)-(8) for each water quality variable,

$$
\begin{gathered}
\frac{\partial}{\partial t}\left(B_{i} H\right)+\frac{\partial\left(u B_{i} H\right)}{\partial x}+\frac{\partial\left(v B_{i} H\right)}{\partial y}+\frac{\partial}{\partial \sigma}\left(w B_{i}\right) \\
=\frac{\partial}{\partial \sigma}\left(\frac{K_{\mathrm{H}}}{H} \frac{\partial B_{i}}{\partial \sigma}\right)+H F_{B_{i}}+R_{B i} \quad i=1, n
\end{gathered}
$$

where $R_{B_{i}}$ represents the reaction, sources and sinks term. The $2 \mathrm{DH}$ models are based on similar transport equations but where only the two horizontal dimensions are considered.

Correct organization, edition and visualization of the large amount of data associated with hydrodynamic and water quality problems are of crucial importance for the correct analysis and interpretation of the scenarios established. Thus, several tools were integrated into the hydroinformatic environment to carry out the pre- and postprocessing tasks, and a conditioned mesh refinement methodology was established. A GIS tool was made available to deal with both the input data and the most relevant model results. Input data and results analysis are essentially performed using the SMS software. This software is used to organize input data and to visualize the model's results. It is capable of representing scalar and vectorial variables. The GIS software used was ARCVIEW, and a computer aided design tool (AUTOCAD) was used to edit and digitalize the graphic images. The mesh generation methodology was implemented by means of the TRIANGLE software [8]. Tidal water surface elevations, used as boundary conditions in most coastal waters models, were calculated with the SR95 model [9]. This software is based on satellite observation data. GRIDGEN and CSLICE are MATLAB based tools developed for the grid generation and results presentation, respectively, of the POM model. A database software tool (Microsoft ACCESS) was used.

Data from different software packages was integrated and exchanged with the aid of a group of utilitarian tools basically developed to perform conversion and writing tasks according to the appropriate file data formats.

\section{Software developments and innovations}

\subsection{Conditioned mesh refinement}

Finite element mesh resolution for hydrodynamics or/and water quality models must be properly established 
according to the purpose of the model implementation. The size of the elements in a mesh must be defined in such a way that the spatial variation of the calculated variables (velocity components, water depth or water quality variables' concentrations) throughout the domain will be reproduced with a reasonable approximation. The mesh must thus show higher resolutions within areas of more intense variable gradients and should show bigger elements for regions where gradients are less severe. On the other hand, it must be stressed that when the mesh resolution increases, requiring the use of more elements in a mesh, the computational time costs increase too, making the modelling tasks difficult. One of the most efficient techniques for generating finite elements meshes is the Delaunay triangulation [8]. There are several algorithms for this kind of triangulation and they all perform equally well: the incremental insertion algorithm, the divide-and-conquer algorithm and the plane-sweep algorithm [10]. The general methodology for mesh generation implemented uses these algorithms. However, finite element meshes used in coastal waters models must observe certain criteria, among which is the rule that they must be composed of non-distorted elements (triangles or quadrilaterals) whose minimum interior angles must be greater than about $20^{\circ}$. Furthermore, it is desirable that, during the mesh generation process, some control on the elements size over the entire domain must be possible. The Rupert [11] algorithm has all these characteristics and is implemented in the software used.

The methodology adopted for the generation of finite element meshes involved the following main phases: defining boundary domain polygonal line, increasing/ decreasing the polygonal line resolution, forced quality Delaunay triangulation, and conditioned mesh refinement.

Initially the domain must be delimited using a polygonal line (which frequently has to be geometrically simplified). In this phase, two properties of the mesh have to be established: the number (or area) and form of the elements. These properties must conform with the hardware/software capabilities and with the required space resolution. When the forced Delaunay triangulation is used, the total number of elements in the mesh is related to the resolution of the boundary polygonal line. A high resolution of the polygonal line will result in an excessive refinement near the boundary and consequently in a high number of elements.

Therefore, special attention has to be paid to the boundary resolution. The average distance between polygonal vertices must be estimated beforehand, and a procedure of refinement or de-refinement has to be followed in order to fit the boundary resolution to the established maximum number of elements in the mesh.

A group of utilitarian programs was created to accomplish these tasks. These programs comprised: a tool for evaluating the geometric characteristics of the initially digitalized boundary polygonal line; a tool for refining the polygonal line by inserting vertices between the original ones, and a tool for de-refinement, by removing vertices according to a previously established maximum distance between polygonal vertices.

Fig. 2 displays three meshes, considering the constraints of a minimum interior angle $\left(20^{\circ}\right)$ and of a maximum elements area $\left(1000 \mathrm{~m}^{2}\right)$, generated using distinct boundary polygonal line resolutions.

The procedure just described guarantees the generation of a mesh with elements that respect the minimum interior angle and the maximum area restrictions. However, in many model implementations, the mesh will have to be generated according to imposed space elements' size variations (local refinements) that are established on the basis of the local gradient of the modelled variables.

The objective of the last phase of the mesh generation procedure is to define a function in the space domain that is related to the maximum elements' size, in order to carry out a conditioned mesh refinement.

Priori or posteriori error estimation techniques are intrinsically associated with the particular numerical method used in a model and become very complicated to implement with the necessary generalization. Therefore, this option for defining the space function to control the elements' size was not used. As an alternative, a simple methodology was established, involving three steps: the first step consists of defining the elements' size control function based on a general criterion (such as water depth, velocity magnitude gradients, pollutant concentration gradients, etc.) and defining the new total number of elements in the mesh; the second step proceeds with computing the maximum area

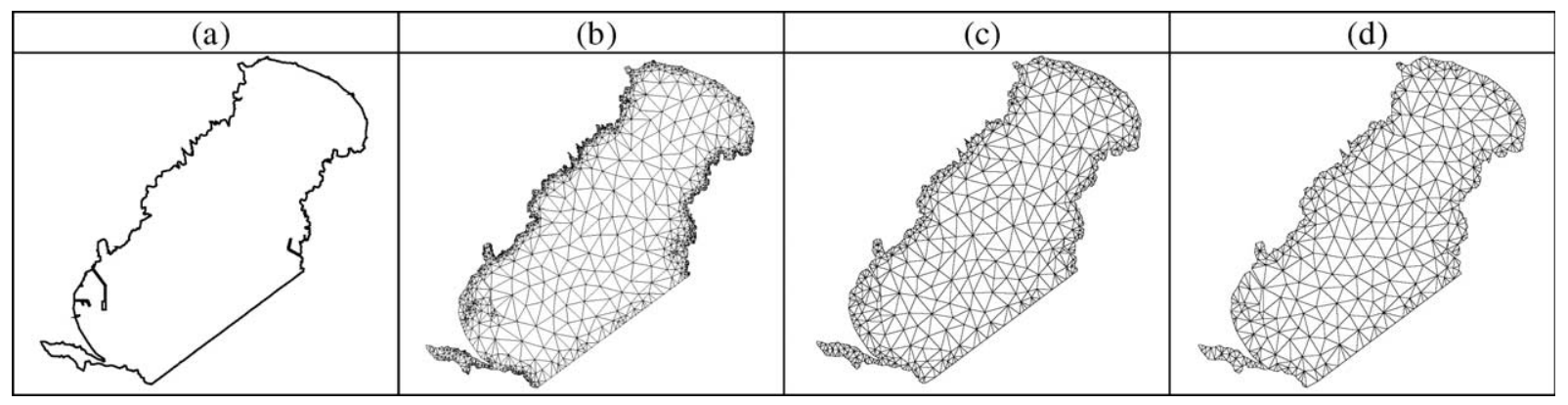

Fig. 2. Meshes generated with different resolutions of the boundary polygonal line. (a) Polygonal line. Average distance between vertices: (b) 24.1 m; (c) $54.7 \mathrm{~m}$; (d) $74.5 \mathrm{~m}$. Total number of triangular elements: (b) 2635; (c) 996; (d) 710. Number of vertices: (b) 1807 ; (c) 709; (d) 503. 


\begin{tabular}{|c|c|c|c|c|}
\hline (a) (c) & (c) & (d) \\
\hline
\end{tabular}

Fig. 3. Finite element meshes for the River Cávado estuary: (a) no minimum angle restriction, maximum area: not considered, elements: 144; (b) minimum angle: $20^{\circ}$, maximum area: not considered, elements: 338 ; (c) minimum angle: $30^{\circ}$, maximum area: not considered, elements: 738 ; (d) minimum angle: $30^{\circ}$, maximum area: $2000 \mathrm{~m}^{2}$, elements: 1572 .

of the elements in the initial mesh, and in the third step, the initial mesh is refined considering the area restrictions computed in the second step.

Fig. 3 presents meshes generated for the River Cávado estuary (north Portugal). The total number of elements increases fivefold when the minimum interior angle ranges from 0 to $30^{\circ}$, as can be seen in the meshes (a) to (c).

Mesh (d) in Fig. 3 was generated considering a minimum interior angle of $30^{\circ}$ and a maximum area of $2000 \mathrm{~m}^{2}$, resulting in a mesh with 1572 elements. This mesh was used in the implementation of a model to study the hydrodynamics and a hypothetical accident involving a pollutant discharge in the estuary. In order to improve the performance of the model two different meshes were used. These meshes were generated considering the conditioned refinement of mesh d) using two distinct criteria: the first one considered the depth gradient as the control function, and in the second one, the control function was established according to the elements' proximity to a fixed point in the interior of the estuary (Fig. 4).
As a complement to the conditioned mesh refinement methodology described above, three different finite elements mesh performance evaluation methods for a hydrodynamic model were developed.

In the first method an index is computed as the sum of products of the element average velocity module at one instant of the simulation (coincident with the maximum velocity instant during ebb tide) times the respective element area. The variation of this index between different meshes makes it possible to evaluate the sensitivity of the computed results to the mesh resolution variation.

The second method consists of velocity module mapping within the model domain (for the same instant as before) considering different velocity module ranges. A comparison of the configurations obtained allows analysis of the sensitivity of the computed results to the meshes' resolutions in spatial terms.

Finally, in the third method, several particles released at different points in the model were considered, and their trajectories are computed using the hydrodynamic results

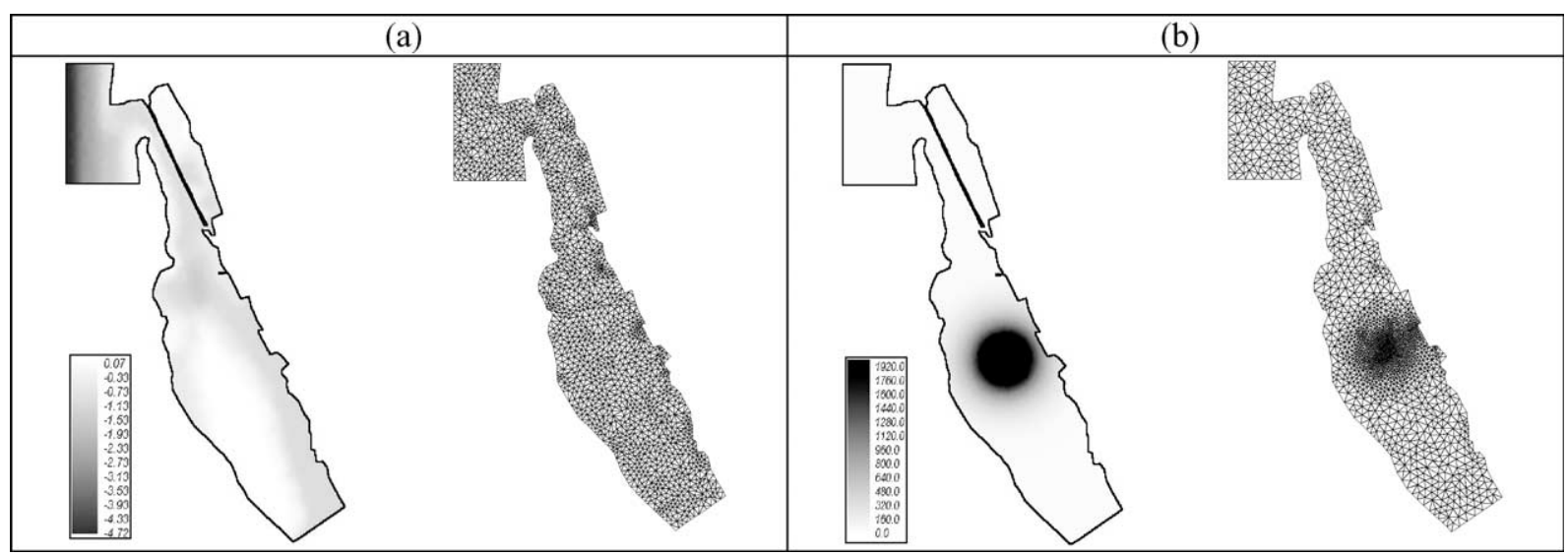

Fig. 4. Conditioned mesh refinement. Control function of the maximum elements areas: (a) depth gradient; (b) proximity to a fixed point in the interior of the estuary. 
obtained with different meshes. Comparisons between the particle displacements obtained with different meshes are used to evaluate the meshes' performance.

These three methods were used to select the most appropriate mesh for the Ria de Arosa (NW Spain) 2DH hydrodynamic model.
Two groups of meshes (Fig. 5) have been generated. In the first group, meshes of increasing resolution have been generated, taking as refinement criteria an internal minimum angle of $30^{\circ}$ and a maximum area restriction established uniformly for the entire domain (meshes AR1 to AR5). In the second group, the meshes have been

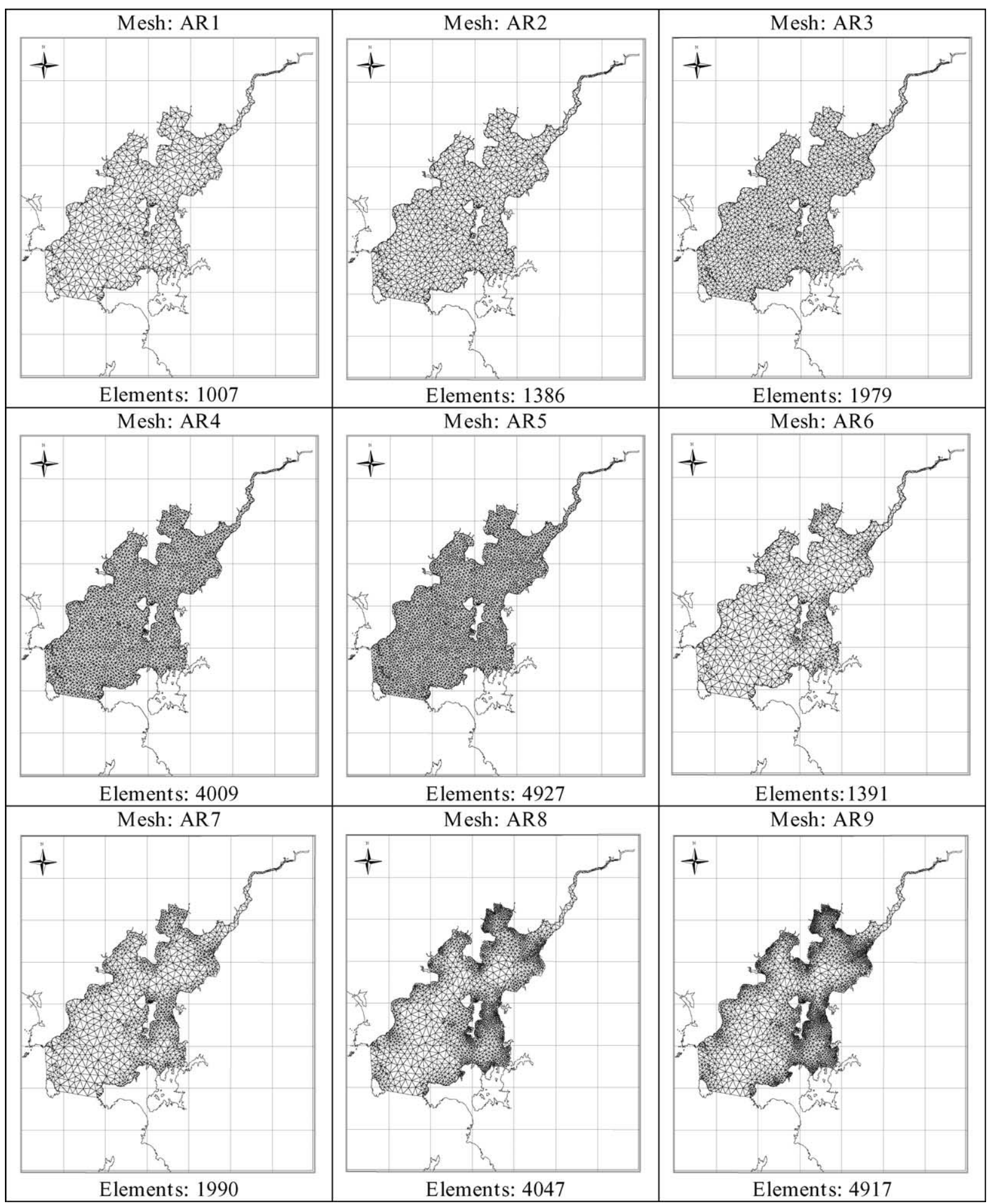

Fig. 5. Finite elements meshes for the Ria de Arosa (NW Spain) 2DH hydrodynamic model. 
generated from the conditional refinement of mesh AR1, with refinement criteria being the imposition of a minimum internal angle $\left(30^{\circ}\right)$ and a maximum area restriction established from a control function dependent on the average depth. This control function was defined in such a way that the refinement occurs preferentially in shallow regions. The number of elements of these meshes (AR6 to AR9) is close to the number of elements of the first group of meshes (AR2 to AR5).

The mesh selection procedure considered the computational costs (CPU simulation time) associated with each mesh. The quotient $(f)$ between the CPU simulation time associated with each mesh and the CPU simulation time of the AR1 mesh was computed, considering a simulation of a semi-diurnal tide during two tide periods (approximately $25 \mathrm{~h}$ ).

Mesh performance indices were compared independently for the two sets of generated meshes, considering a tide simulation (graphs (a) and (b)-Fig. 6) and a simulation considering the tide and the wind acting simultaneously (graphs (c) and (d)-Fig. 6). For each group the computed value for the greater resolution mesh was used as a reference value for comparison purposes.

The relative variation of the performance indices is equal or inferior to $2.1 \%$ for all comparisons. A strong reduction is observed when the number of elements goes up from 1000 (mesh AR1) to 1400 (meshes AR2 or AR6). The results obtained are less sensitive to the variation of the number of elements from 1400 to 2000 (meshes AR3 and AR7) and to 4000 (meshes AR4 and AR8). The CPU simulation times associated with these two last meshes are, however, about 30 times superior to the CPU simulation time of mesh AR1.

In order to evaluate the results' sensitivity to the meshes' resolution, in spatial terms, the second method, proposed above, for the mesh performance evaluation was adopted. The configurations of the resultant areas, considering five velocity modules intervals (at the instant of the maximum ebb tide velocity occurrence), have thus been mapped using a GIS tool. As can be seen in Fig. 7, for meshes AR4 and AR5 (included in the first group of meshes), the mapping of the computed velocity modules leads to similar configurations. For the second group of meshes, presented in Fig. 8, the resulting configuration for the mesh AR7 is close to the configurations obtained with meshes AR8 and AR9. However, the f quotient for the AR7 mesh is 3 while for the other two meshes the same quotient increases to 29 and 74 , respectively.

The third method proposed for mesh performance evaluation was applied considering a release of ten particles. Their trajectories (Fig. 9) were computed for a hydrodynamic simulation considering the tide and the wind action during a $12.5 \mathrm{~h}$ period. Trajectories showing a bigger sensitivity to the variation of the meshes' resolution are the $\mathrm{T} 2, \mathrm{~T} 3, \mathrm{~T} 5, \mathrm{~T} 7$ and $\mathrm{T} 10$ trajectories, which correspond to particles that have been released at points located in shallower regions (water depth lower than $10 \mathrm{~m}$ ). Remaining particles have been released at points where depths are greater than $20 \mathrm{~m}$.

In order to compare the meshes' performance, both the distances between the final particle positions for each mesh
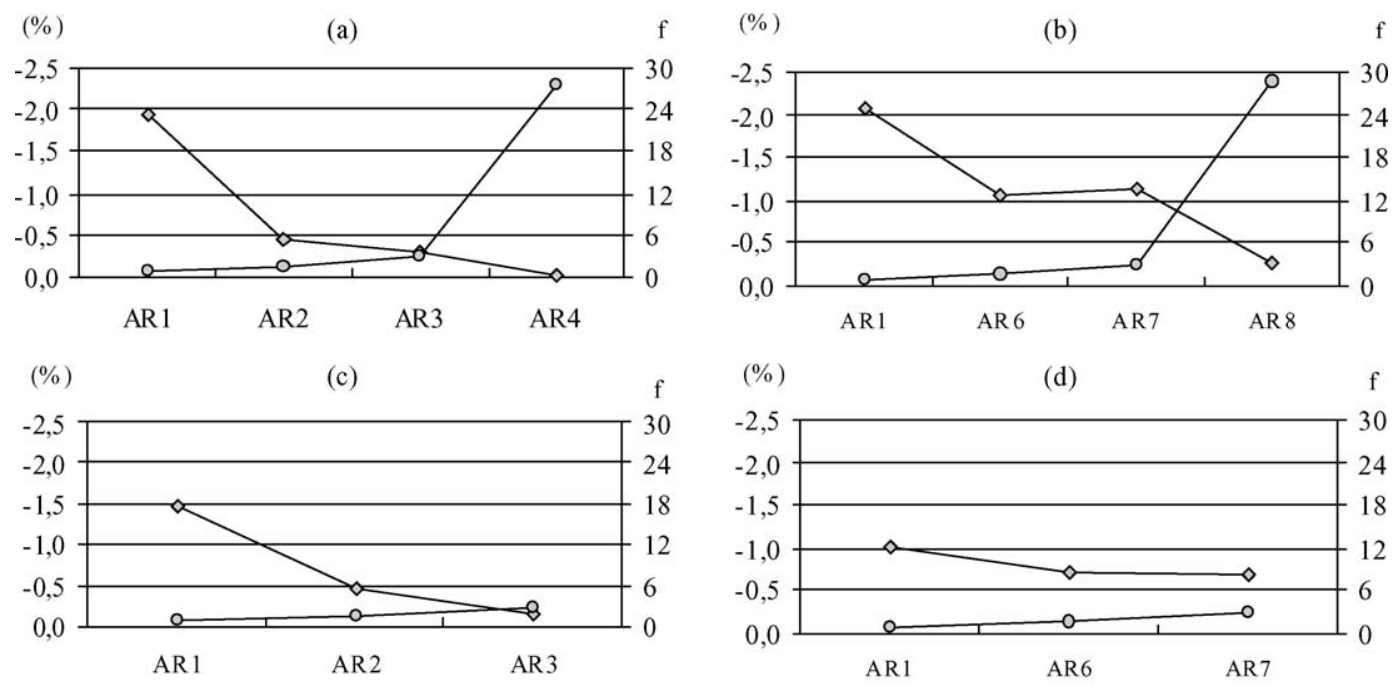

\begin{tabular}{|l|l|}
\hline & $\begin{array}{l}\text { Quotient (f) between the CPU simulation time of each mesh and the CPU } \\
\text { simulation time of AR1 mesh. }\end{array}$ \\
\hline & $\begin{array}{l}\text { Relative variation of the performance indice considering as reference value: a) } \\
\text { AR5 indice; b) AR9 indice; c) AR4 indice and d) AR8 indice. }\end{array}$ \\
\hline
\end{tabular}

Fig. 6. Relative differences of the mesh performance index and f quotients for hydrodynamic simulations using different meshes: (a) and (b) tide action; (c) and (d) tide and wind acting simultaneously. 


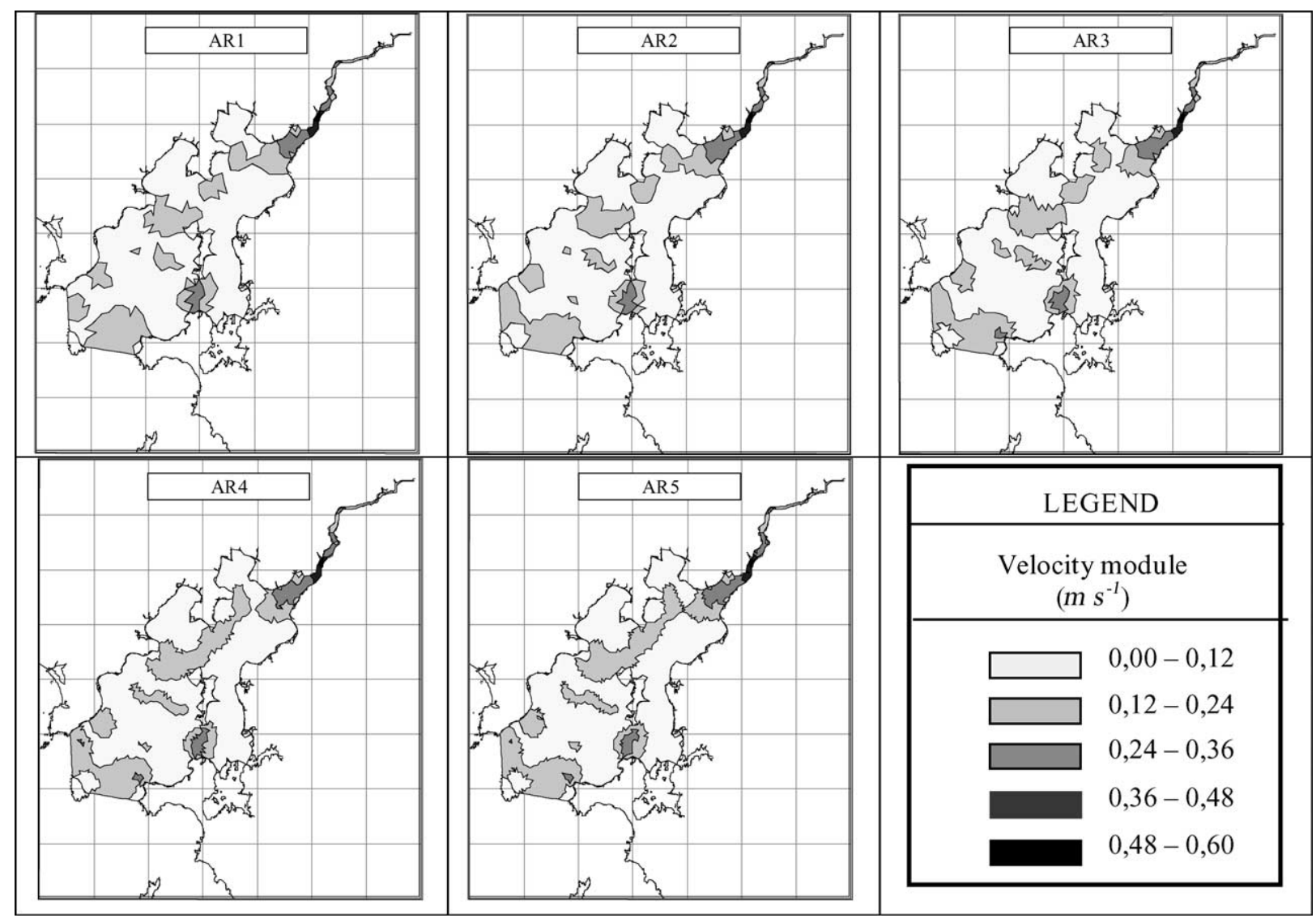

Fig. 7. Maximum velocity module for the ebb tide computed using meshes AR1, AR2, AR3, AR4 and AR5.

were computed, and the final particle positions obtained with the higher resolution meshes (AR4 and AR8). The sum of these distances (Fig. 10) is lower for meshes AR6 and AR7, when compared with the corresponding total distances for meshes AR2 and AR3. The present method of mesh performance evaluation shows that the conditional mesh refinement using the depth criterion (meshes AR6 to AR9) leads to better results than the mesh refinement that takes a uniform distribution of elements.

The performance analysis carried out using a global index reveals greater sensitivity in the results for the AR6 to AR8 group of meshes. However, the relative variation of this performance index is less than $1.0 \%$ for the two hydrodynamic simulations considered (while that in the AR2 to AR4 group of meshes is below $0.5 \%$ ).

Mapping the velocity module provides configurations that are nearest to the ones obtained with the higher resolution meshes (meshes AR5 and AR9) in the AR6 to AR8 group of meshes.

This group also reveals a better performance for the trajectory performance evaluation method. In fact, it is in shallow regions that water currents will suffer a bigger influence from the wind action (analysed hydrodynamic situation). In the Ria de Arosa hydrodynamic model the AR7 mesh was adopted. This mesh performs satisfactorily when compared with meshes of higher resolution, and, furthermore, it is not very demanding in terms of CPU simulation time (equal to three times the CPU simulation time of mesh AR1).

After calibration and validation the model was used to study the principal characteristics of the water currents in Ria de Arosa. Some of the results obtained are given below. The principal currents in the Ria are mainly caused by tide action $[12,13]$. Several simulations were carried out to quantify tide currents within the Ria. Fig. 11 shows the maximum velocity fields for a spring tide $(3.75 \mathrm{~m}$ tide height) and for a neap tide ( $1.5 \mathrm{~m}$ tide height).

For the spring tide, the maximum velocity reaches values of $0.5 \mathrm{~ms}^{-1}$ in three different regions: the river Ulla estuary; a zone located between the Arosa island and the El Grove peninsula, and a region close to the ocean open boundary north of Sálvora island. Tide current velocities present values ranging from 0.25 to $0.30 \mathrm{~ms}^{-1}$ in the principal channel, in the central part of the Ria, a region located northwest of Arosa island. Tide current velocities in the Puebla del Caramiñal inlet and in a limited region east of Arosa island are well known.

In the present study, various simulations, considering different wind directions and intensities, were carried out in order to characterize the vertically averaged wind current patterns. The most frequently occurring summer and winter wind conditions were considered. For summer the wind 


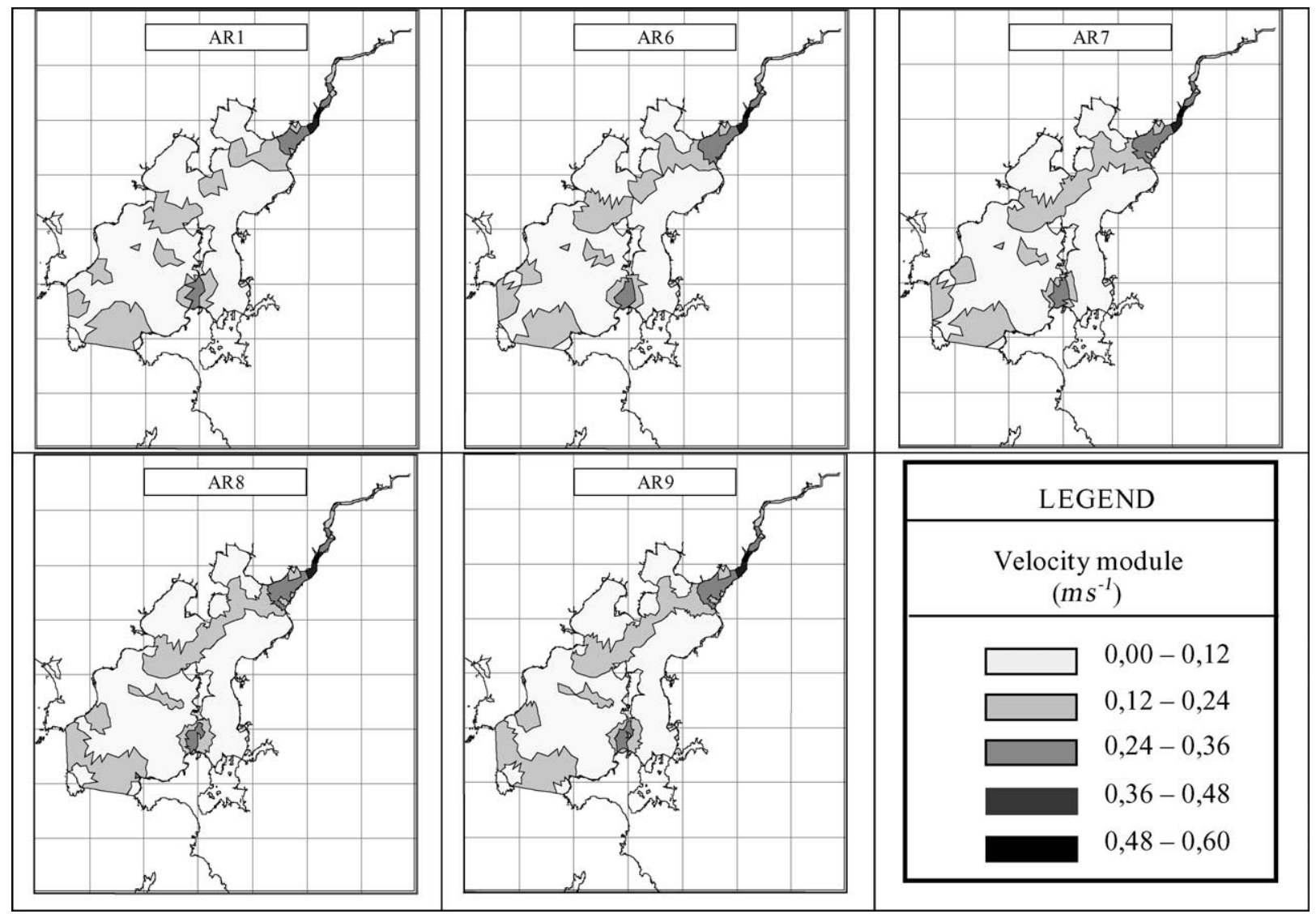

Fig. 8. Maximum velocity module for the ebb tide computed using meshes AR1, AR6, AR7, AR8 and AR9.

blowing from the northern quadrant was taken, as this is the commonest wind direction for this season, with a permanent mean velocity of $5 \mathrm{~m} \mathrm{~s}^{-1}$, while for winter the wind blowing from the southern quadrant was considered, with a permanent mean velocity of $3 \mathrm{~m} \mathrm{~s}^{-1}$.

Fig. 12 shows the vertically averaged wind velocity results for these summer and winter wind conditions. The regions most sensitive to wind action are, as expected, the more shallow regions: almost all the areas around Arosa island and the inner part of the Ria close to the river Ulla estuary.

Presented results show two current gyres, one in the internal part of the Ria and the other at the central part, including the southeast region of Arosa island, with a clockwise rotation for northern winds and a counter-clockwise rotation for southern winds. Maximum wind current velocities ranges, for summer situation, from $6 \mathrm{~cm} \mathrm{~s}^{-1}$ (west of Arosa island) to $15 \mathrm{~cm} \mathrm{~s}^{-1}$ (east of Arosa island). Currents are reversed when the wind direction changes from north to south.

River discharges (Ulla and Umia rivers) have a local effect in the current patterns of the Ria. Really, considering only the river discharges into the Ria (neglecting tide and wind currents) various simulations were worked out and have shown that its effect in the current patterns is restricted to the rivers mouth. Maximum velocities are 35

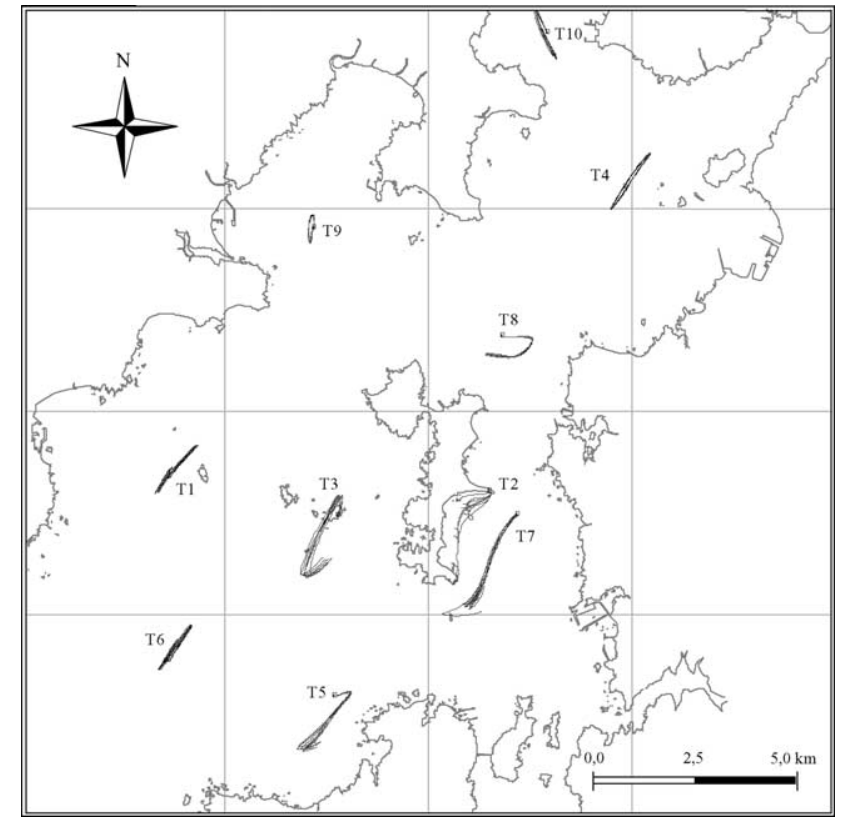

Fig. 9. Particle trajectories computed for the hydrodynamic simulations under the tide and the wind action using meshes AR1, AR2, AR3, AR4, AR6, AR7 and AR8. 

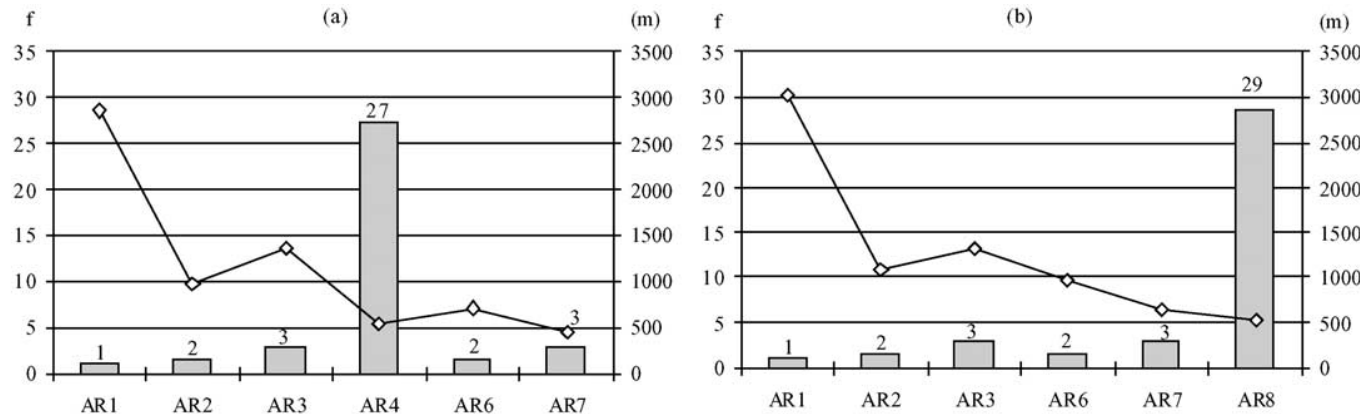

\begin{tabular}{|l|l|}
\hline & $\begin{array}{l}\text { Quotient (f) between the CPU simulation time of each mesh and the CPU simulation time of } \\
\text { AR1 mesh. }\end{array}$ \\
\hline & $\begin{array}{l}\text { Sum of distances between final particles positions for each mesh and those correspondents to: } \\
\text { a) AR8 mesh; b) AR4 mesh. }\end{array}$ \\
\hline
\end{tabular}

Fig. 10. Sum of the distances between the final positions of the particles for each mesh and the final positions for meshes AR8 (a) and AR4 (b).

and $2 \mathrm{~cm} \mathrm{~s}^{-1}$ for the river Ulla and river Umia, respectively, considering the winter discharges of these rivers. Fig. 13 presents current velocities in the Ria considering different tide water levels.

\subsection{POM-UMH model}

The POM-UMH model is a modified version of the POM model. POM is a sigma coordinate model in that the vertical coordinate is scaled on the water column depth; the horizontal grid uses curvilinear orthogonal coordinates and an 'Arakawa C' differencing scheme; the horizontal time differencing is explicit, whereas the vertical differencing is implicit; it contains an imbedded second moment turbulence closure sub-model to provide vertical mixing coefficients; it uses the Smagorinsky diffusivity for horizontal diffusion, although a constant or biharmonic diffusion can be used instead; it has a free surface and a split time step. The external mode portion of the model is twodimensional and uses a short time step based on the CFL condition and the external wave speed. The continuity and momentum equations solved in the external mode have the following forms:

$$
\begin{aligned}
& \frac{\partial \eta}{\partial t}+\frac{\partial U H}{\partial x}+\frac{\partial V H}{\partial y}=0 \\
& \frac{\partial U H}{\partial t}+\frac{\partial U^{2} H}{\partial x}+\frac{\partial U V H}{\partial y}-\tilde{F}_{x}-f V H+g H \frac{\partial \eta}{\partial x} \\
& =-\langle w u(0)\rangle+\langle w u(-1)\rangle+G_{x} \\
& \quad-\frac{g H}{\rho_{0}} \int_{-1}^{0} \int_{\sigma}^{0}\left[H \frac{\partial \rho}{\partial x}-\frac{\partial H}{\partial x} \overline{\bar{\sigma}} \frac{\partial \rho}{\partial \bar{\sigma}}\right] \mathrm{d} \bar{\sigma} \mathrm{d} \sigma
\end{aligned}
$$

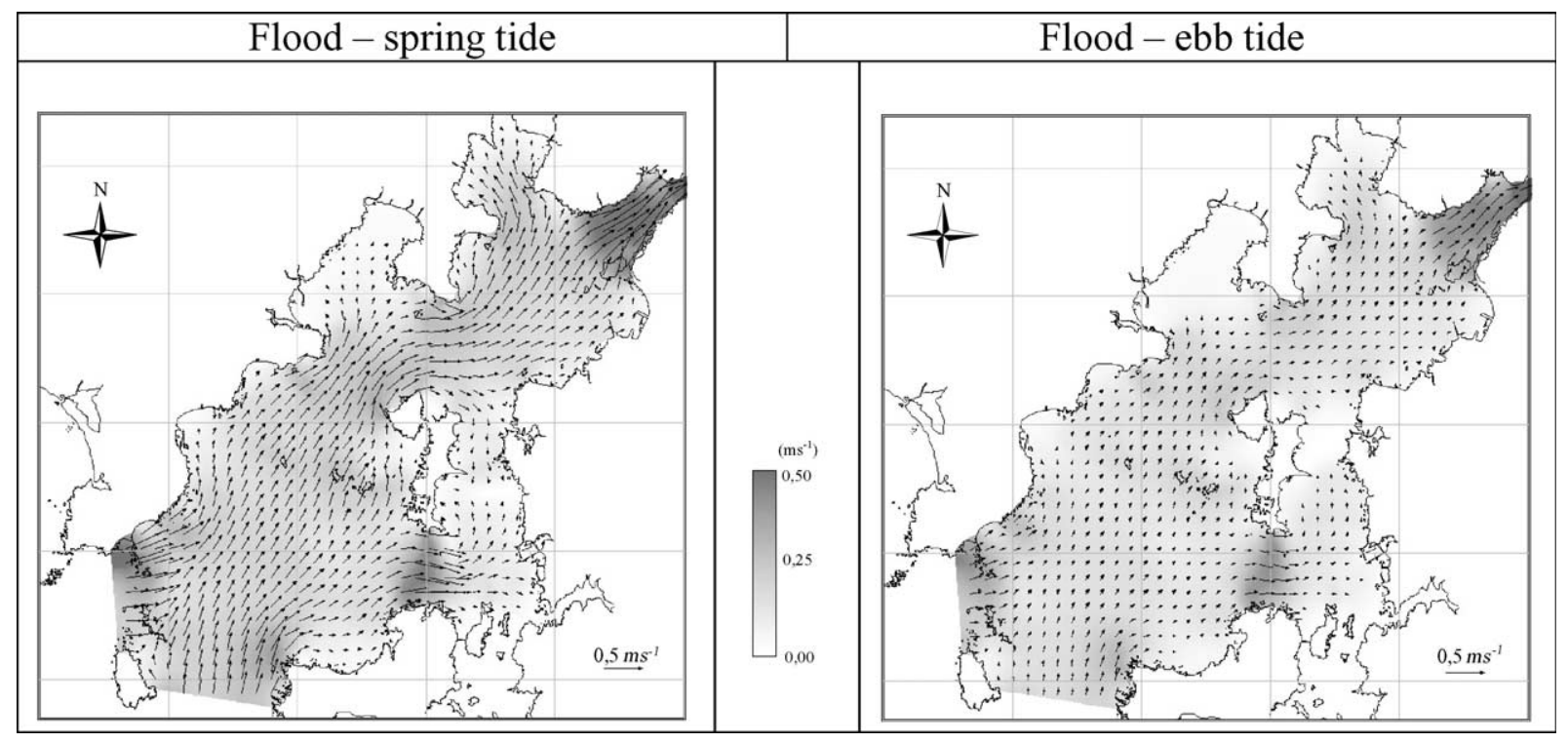

Fig. 11. 2DH Ria de Arosa hydrodynamic model: maximum flood tide current velocities for a spring and a neap tide. 


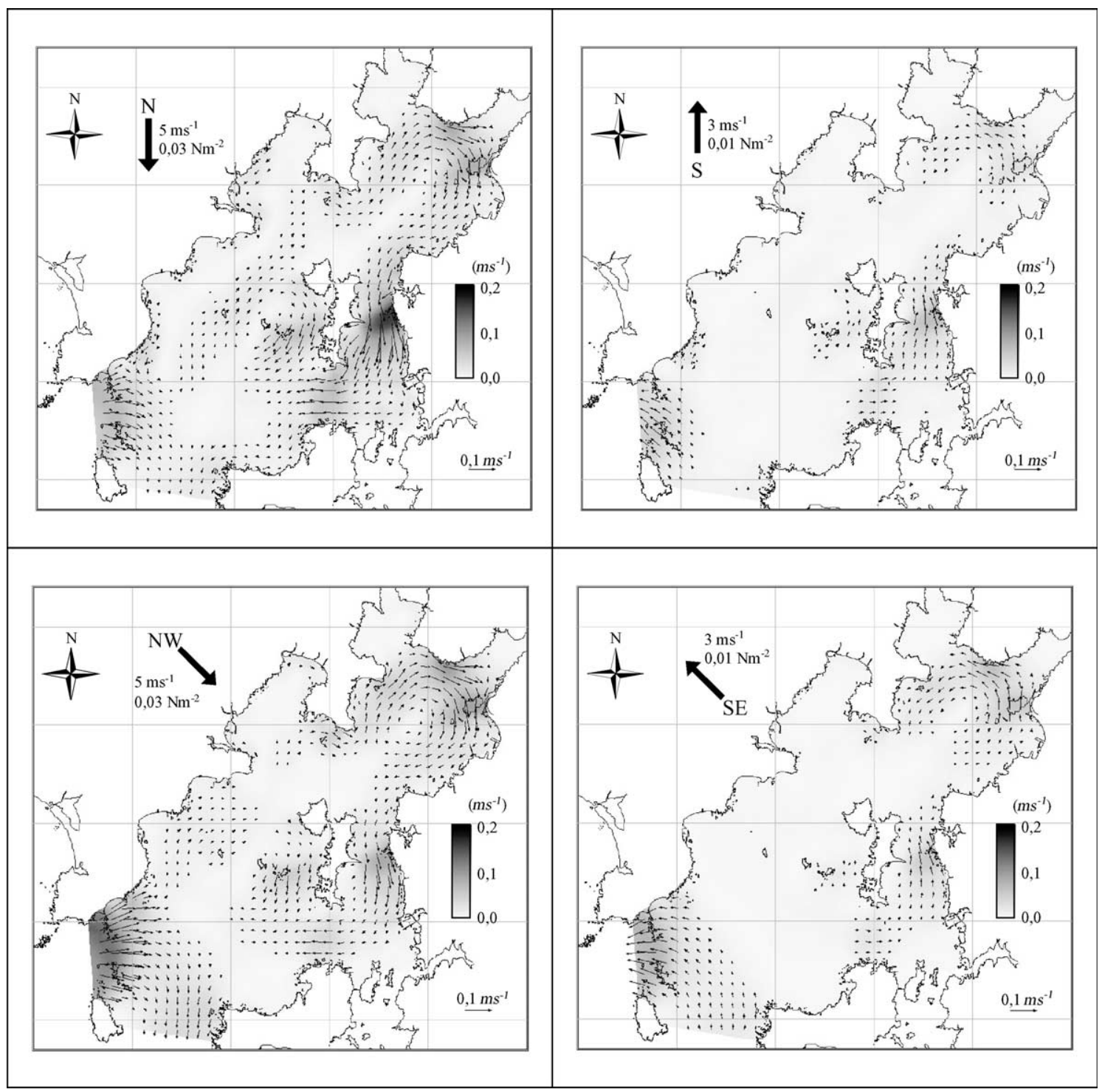

Fig. 12. 2DH Ria de Arosa hydrodynamic model: wind current velocities for the most frequent winter and summer wind directions.

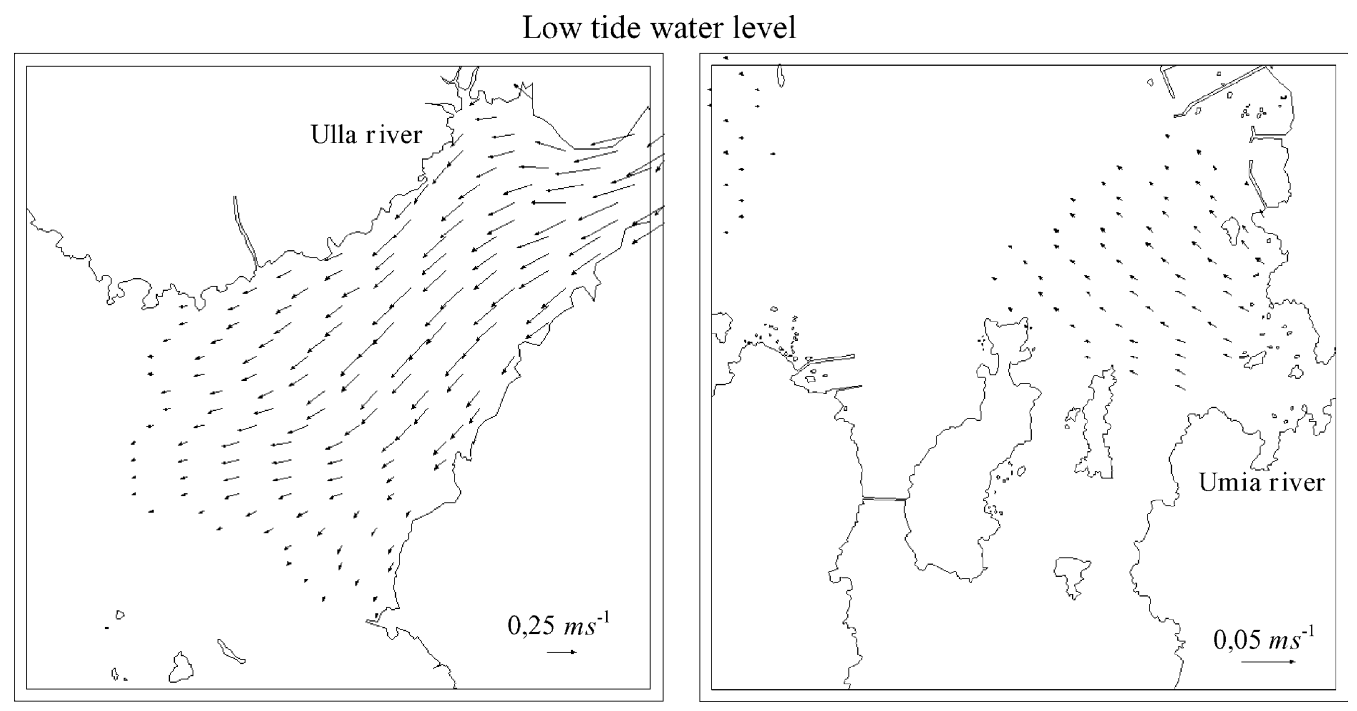

Fig. 13. 2DH Ria de Arosa hydrodynamic model: river discharges' current velocities for different Ria water levels. 


$$
\begin{aligned}
\frac{\partial V H}{\partial t} & +\frac{\partial U V H}{\partial x}+\frac{\partial V^{2} H}{\partial y}-\tilde{F}_{y}+f U H+g H \frac{\partial \eta}{\partial y} \\
= & -\langle w v(0)\rangle+\langle w v(-1)\rangle+G_{y} \\
& -\frac{g H}{\rho_{0}} \int_{-1}^{0} \int_{\sigma}^{0}\left[H \frac{\partial \rho}{\partial y}-\frac{\partial H}{\partial y} \bar{\sigma} \frac{\partial \rho}{\partial \bar{\sigma}}\right] \mathrm{d} \bar{\sigma} \mathrm{d} \sigma
\end{aligned}
$$

The terms $\langle w u(0)\rangle$ and $\langle w v(0)\rangle$ are the surface wind stress components and $\langle w u(-1)$ and $w v(-1)\rangle$ are the bottom stress components. $\tilde{F}_{x}$ and $\tilde{F}_{y}$ are the horizontal diffusion terms and $G_{x}$ and $G_{y}$ are the dispersion terms. The internal mode is $3 \mathrm{D}$ and uses a long time step based on the CFL condition and the internal wave speed.

In hydrodynamic studies using mathematical modelling, boundary conditions at open boundaries are preferably defined using water velocity components. However, in most case studies, it is easier to obtain measurements of the surface elevations than measurements of water current velocities. Thus, the performance of a model will depend on a number of factors, including the performance of the program when open boundary conditions are imposed, considering water level records. On the other hand, finite elements mesh based models are more suitable for applications involving geometrically complex problems than finite differences grids.

To evaluate the performance of the external mode of the POM model and the RMA2 finite elements model, two hydrodynamic simulations were carried out considering the open boundary conditions imposed by water surface elevations. The comparison was made in order to evaluate the expected differences between the two different numerical models.

The test case assumed a rectangular basin with an open boundary, which is imposed with a water surface elevation sinusoidal function. This hydrodynamic test case presents an analytical solution under specific simulation conditions that were adopted in the models implemented [3]. The POM model was created using a $25.0 \mathrm{~m}$ constant finite differences grid, and the finite elements model was implemented using a mesh with 246 quadratic quadrangular elements.

A 1 s time step was taken for the POM model and a 25 s time step adopted for the RMA2 model. The water is assumed to be at rest at the beginning of the simulation, and a total simulation time equal to six times the wave period $(6000 \mathrm{~s})$, imposed at the open boundary, was considered. The findings at the middle node are presented in Fig. 14.

Results presented previously for the two model simulations reveal that the RMA2 model performs better. Comparing the sums of the velocity differences module for the numerical and analytical solutions, we find that the sum given by the RMA2 model results is about $30 \%$ less (considering the entire simulation period). This model also presents a minor transient period between the initial condition and the dynamic solution, as can be seen in Fig. 14. These results are consistent with the characteristics of the numerical techniques used for each model [14].

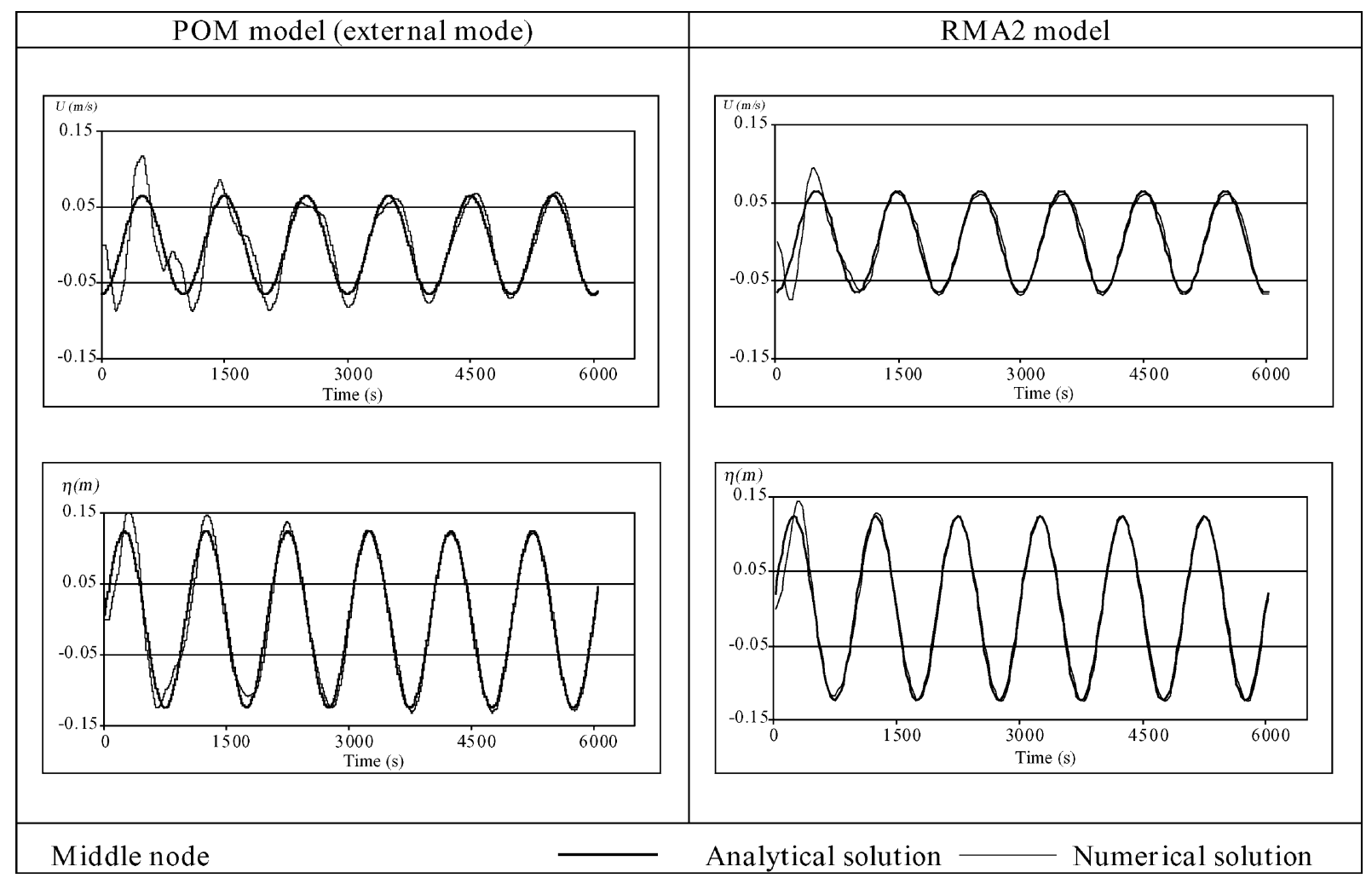

Fig. 14. Analytical and numerical solutions (POM model and RMA2 model) at the middle node of the test case. 
As mentioned earlier, the substitution of the external mode technique calculation in the original POM version gives rise to the POM-UMH model. The present version of the program is valid for applications where the baroclinic terms (involving vertical density gradients) and the dispersion terms $G_{x}$ and $G_{y}$ (Eqs. (15) and (16)) can be ignored. The time step is limited by the CFL condition associated with the internal mode, since the implicit numerical technique used in the external mode does not present a time step restriction. The formulations used for surface and bottom stress computations are similar for both modes. Vertical averages of the viscosity coefficients computed in the internal mode, as well as the bottom stresses, are transferred to the external mode at every time step. The variable value transfer between the internal mode grid and the external mode mesh is carried out by means of appropriate pointers, which are defined such that they associate each node of the mesh with a node of the grid.

The associated cost of the external mode substitution in the original POM model is an increase in the computer time needed to perform a simulation. In order to evaluate this computational time several quasi-3D hydrodynamic simulations were carried out, considering a rectangular basin (dimensions $30 \mathrm{~km} \times 10 \mathrm{~km}$ ) with a $20 \mathrm{~m}$ constant depth and with an open boundary imposed by a dynamic water surface elevation condition. A different spatial resolution was used for each simulation: in the POM model, equally spaced grids were used in the horizontal plane, since, in the RMA2 models, meshes formed by quadratic quadrilateral elements of the same size as the grid cells were implemented. The computational times obtained (for a PC computer with a Pentium $500 \mathrm{MHz}$ processor) are presented in Table 1.

The computational time increase depends on the grid spatial resolution. It varies from $19 \%$ for a grid with $61 \times 21$ divisions in the horizontal plane and 21 divisions in the vertical plane, to about four times greater for a grid with $121 \times 41$ horizontal divisions and with three divisions in the water column. However, this increase can be mitigated if a low-resolution mesh is used for the external mode computations. In this case, the variables required for the internal mode (computed in the external mode) can be obtained by interpolating the values computed in the finite elements mesh. To illustrate the POM-UMH program potential for the implementation of models that only need a 3D computation of the hydrodynamic water current features in sub-regions of the entire domain, an example involving two basins linked by a narrow channel was worked out (Fig. 15). The left basin has an open boundary that was imposed by a sinusoidal water surface elevation condition. In the right basin, there is a bottom elevation singularity. The 3D features of the induced flow were computed for this region using a sub-model grid with 10 divisions for both horizontal directions and 20 layers in the vertical direction. Only the internal mode is computed within this region. Open boundary conditions for the internal mode were established according to the external mode results computed.

Fig. 16 gives the flow field for the instant of maximum flood velocities. These results were processed using the SMS program and other tools created for post-processing purposes.

\subsection{Water quality models: PROCESSES, RMA4-UMQ and POM-UMQ}

Simultaneous modelling of hydrodynamics and water quality in coastal zones requires the development of a common structure that allows the resolution of the equations representing the physical water behaviour (conservation of mass and momentum equations), and mass transport equations (advection-diffusion-reaction equations of water dissolved substances). This last set of equations permits to characterize the dynamic distribution of water quality variables that can be used as indicators of the water quality status. With respect to the first set of equations, the mathematical formulations are almost unanimously accepted (with exception for some turbulence modelling aspects). Formulations developed for water quality process modelling are not as consensual since drastic simplifications are used because there are no universal laws for the water quality indicators reactions. Thus, the establishment of mathematical

Table 1

POM and POM-UMH computational time for different spatial resolutions

\begin{tabular}{|c|c|c|c|c|c|}
\hline $\begin{array}{l}\text { Grid divisions } \\
X \text { direction IM }\end{array}$ & $\begin{array}{l}\text { Grid divisions } \\
Y \text { direction } \mathrm{JM}\end{array}$ & $\begin{array}{l}\text { Grid divisions } \\
Z \text { direction } \mathrm{KB}\end{array}$ & $\begin{array}{l}\text { Computation time POM } \\
\text { (1) min/one day simulation }\end{array}$ & $\begin{array}{l}\text { Computation time POM-UMH } \\
\text { (2) } \mathrm{min} / \text { one day simulation }\end{array}$ & {$[(2)-(1)] /(1)$} \\
\hline 121 & 41 & 5 & 48.0 & 152.0 & 2.17 \\
\hline 121 & 41 & 7 & 64.0 & 168.0 & 1.63 \\
\hline 121 & 41 & 11 & 108.0 & 212.0 & 0.96 \\
\hline 121 & 41 & 21 & 270.0 & 374.0 & 0.39 \\
\hline 61 & 21 & 3 & 2.6 & 7.2 & 1.77 \\
\hline 61 & 21 & 5 & 4.0 & 8.6 & 1.15 \\
\hline 61 & 21 & 7 & 7.0 & 11.6 & 0.66 \\
\hline 61 & 21 & 11 & 12.0 & 16.6 & 0.38 \\
\hline 61 & 21 & 21 & 24.0 & 28.6 & 0.19 \\
\hline
\end{tabular}



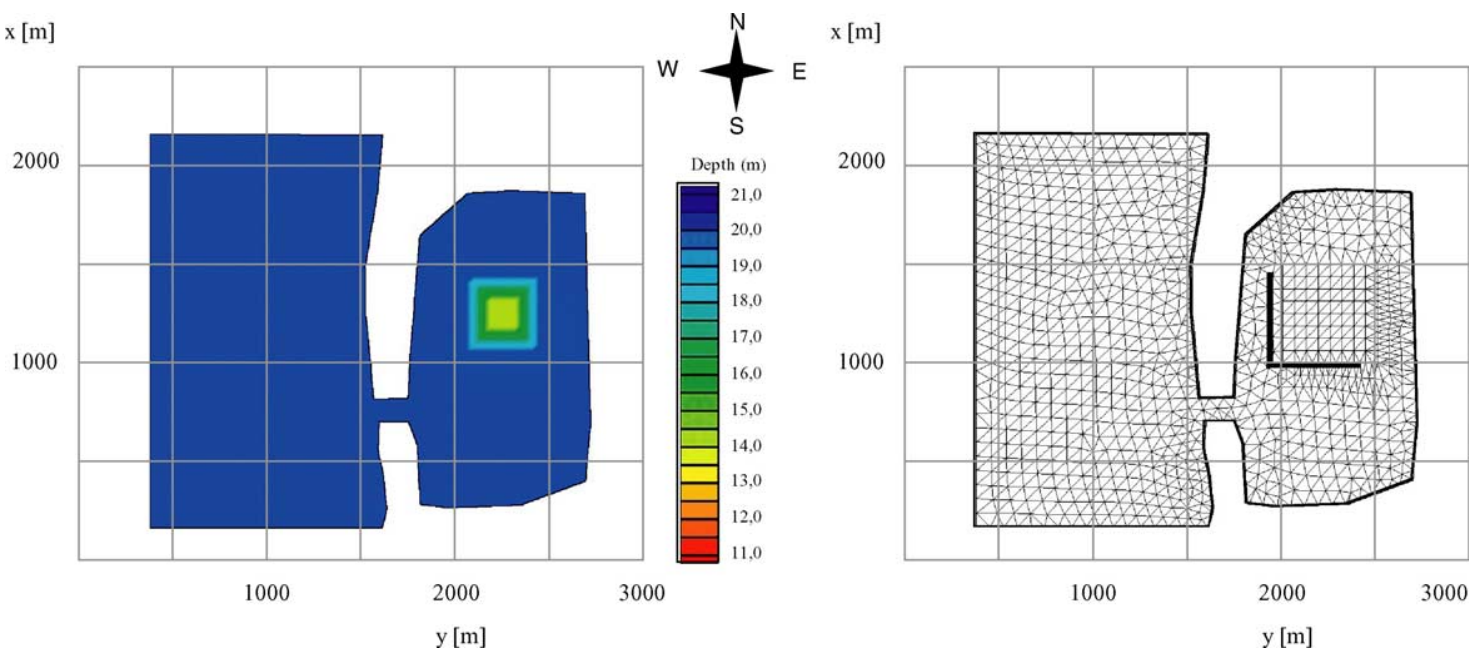

Fig. 15. Example of the POM-UMH model application: geometry and external mode mesh.

formulations to characterize water quality processes (reactions) must always be questioned, and, if possible, validated using field and laboratory data. The commonest water quality process formulations consist of a system of differential equations resulting from considering the mass conservation of a group of substances, which are held to be the most significant for the water quality process. Characterizing the water quality process by mathematical modelling consists of establishing the equations and integrating the resulting differential equations system. A PROCESSES program was developed for this purpose, and this can deal with generic user defined processes and with some of the most commonly occurring water quality processes for coastal water quality studies: conservative and non-conservative

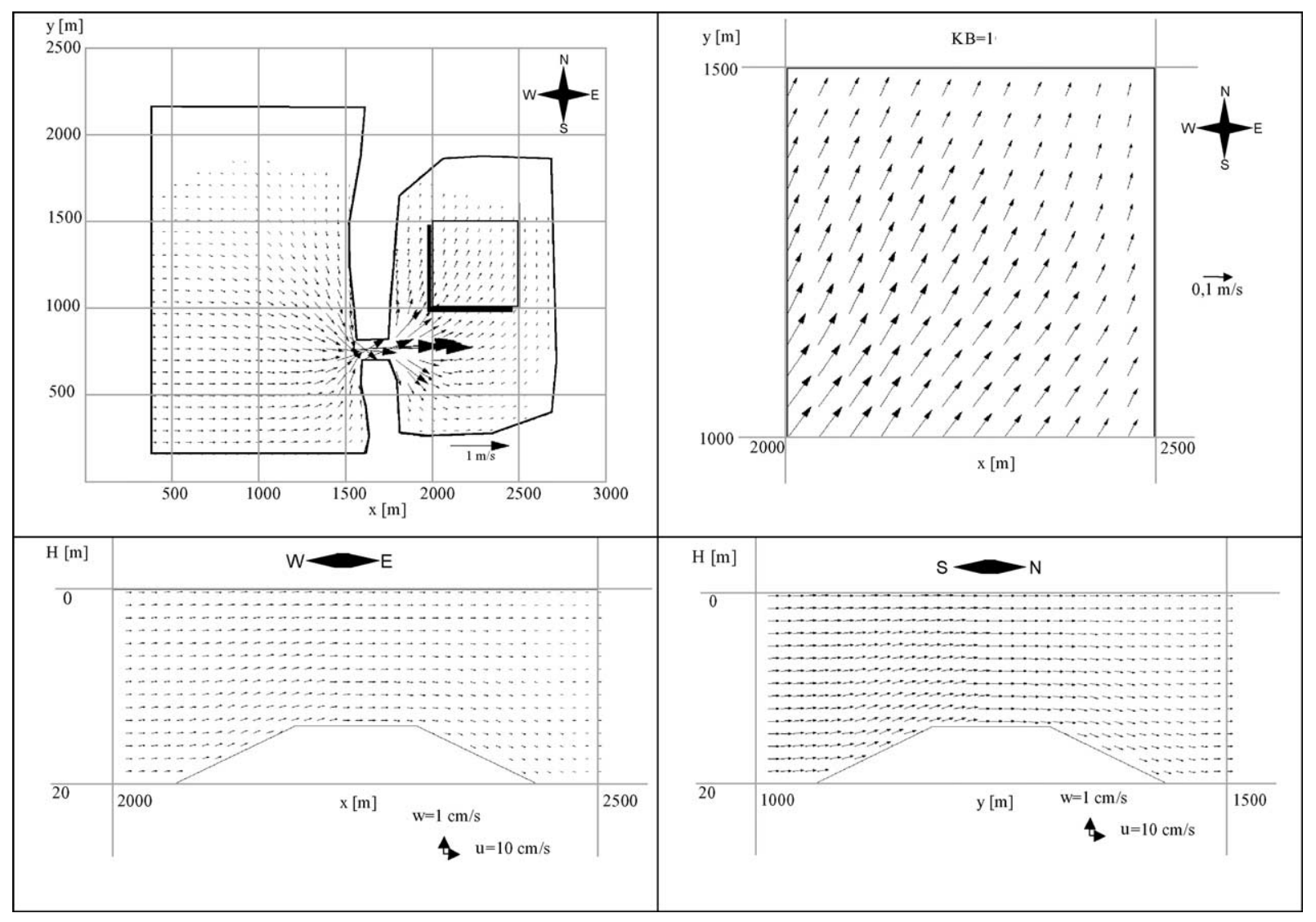

Fig. 16. Example of the POM-UMH model application: results for the instant of maximum flood velocities. 
Table 2

Phytoplankton and herbivorous zooplankton interaction: parameter values for the application of the PROCESSES program

\begin{tabular}{lll}
\hline Parameter & Meaning & Value \\
\hline$a_{0}$ & Initial algae concentration & $1 \mathrm{mgChla} \mathrm{m}^{-3}$ \\
$Z_{0}$ & Initial herbivorous & $0.05 \mathrm{gC} \mathrm{m}^{-3}$ \\
& zooplankton concentration & \\
$a_{\mathrm{ca}}$ & Phytoplankton to carbon ratio & $0.04 \mathrm{gC} \mathrm{mgChla}^{-1}$ \\
$C_{\mathrm{g} z}$ & Grazing rate & $1.5 \mathrm{~m}^{3} \mathrm{gC}^{-1} \mathrm{dia}^{-1}$ \\
$\varepsilon$ & Grazing efficiency & 0.6 \\
$k_{\mathrm{g}}-k_{\mathrm{r} a}$ & Phytoplankton growth rate & $0.3 \mathrm{dia}^{-1}$ \\
& minus phytoplankton respiration rate & \\
$k_{\mathrm{d} z}$ & Herbivorous zooplankton mortality rate & $0.1 \mathrm{dia}^{-1}$ \\
\hline
\end{tabular}

substances; dissolved oxygen, and a primary production process. It uses a numerical integration procedure based on the fourth order Runge-Kutta technique. To illustrate how the PROCESSES program may be applied, a simplified foodchain model involving two variables is considered: phytoplankton concentration $(a)$ and herbivorous zooplankton $\left(z_{h}\right)$ concentration. The mass balance equations for these two water quality variables can be written as follows:

$$
\begin{aligned}
\frac{\mathrm{d} a}{\mathrm{~d} t} & =\left(k_{\mathrm{g}}-k_{\mathrm{ra}}\right) a-C_{\mathrm{g} z} z_{\mathrm{h}} a \\
\frac{\mathrm{d} z_{\mathrm{h}}}{\mathrm{d} t} & =\left(a_{\mathrm{ca}} \varepsilon C_{\mathrm{g} z}\right) z_{\mathrm{h}}-k_{\mathrm{d} z} z_{\mathrm{h}}
\end{aligned}
$$

where all the variables and parameters used have the meanings and the values given in Table 2 .

Results obtained for the phytoplankton and herbivorous zooplankton concentrations (both expressed in $\mathrm{mgC}^{-1}$, to enable its comparison with the total biomass), for an 80 day period, are presented in Fig. 17.

The principal developments introduced in the RMA4UMQ program are the possibility of modelling more than six water quality variables (the original version is limited to six water quality constituents) and the capacity to model water quality processes (reactions). This last development was made possible by introducing a specific subroutine (similar to the PROCESSES program) where the constituent reactions are established beforehand. Thus, between any two time steps (or a multiple period of the time step used) of the diffusion and advection computations, the constituents' decay or growth is computed according to the defined reactions. These computations employ a numerical integration technique similar to that used in the PROCESSES program.

The POM-UMQ program was developed to enable the simultaneous resolution of $3 \mathrm{D}$ hydrodynamics and water quality modelling processes for coastal water systems. The main developments consisted of including an extra transport equation for each of the water quality variables considered in the modelled process and including a subroutine that allows consideration of reactions between constituents that have been added to the set of the model's variables. The numerical method used to solve the new set of equations is analogous to that used by the model for solving the temperature and salinity equations, which are included in the original version of the program. This program can be used to study a diversified set of water quality problems in coastal waters, like wastewater discharge impacts or vulnerability to eutrophication. An example of the POMUMQ application for a submarine outfall impact study is given below (Fig. 18(a)).

Several hydrodynamic scenarios were examined, according to the predominant alongshore currents and tide action, acting either independently or simultaneously. Total coliform bacteria concentration was chosen as the water quality indicator. A non-conservative behavior for this state variable was assumed, which was approximated by a first order decay law. Decay rate for coliform bacteria is highly dependent on the environmental conditions in the receiving water [15]. Thus two different values were taken: 1 and 10 day $^{-1}$. Wastewater discharge was simulated imposing a constant coliform bacteria concentration close to the outfall diffuser (after the initial dilution) equal to 10,000 MPN/100 ml. Resultant outfall plumes for different coliform bacteria decay coefficients, for a permanent South-North alongshore current, can be observed in Figs. 18(b) and 19.

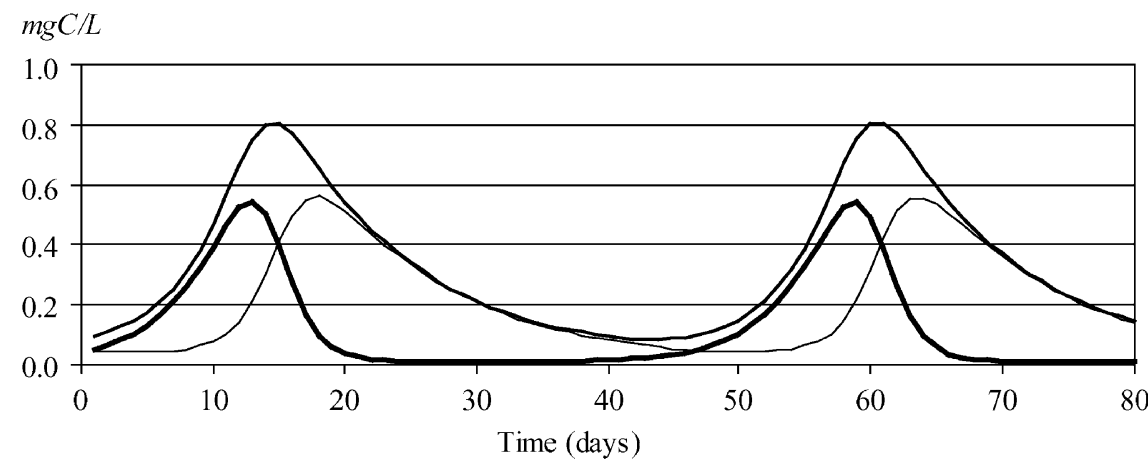

\begin{tabular}{lll|}
\hline Phytoplankton $\quad$ Terb. zooplankton $\quad$ Total \\
\hline
\end{tabular}

Fig. 17. Phytoplankton and herbivorous zooplankton interaction: results computed by the PROCESSES program. 


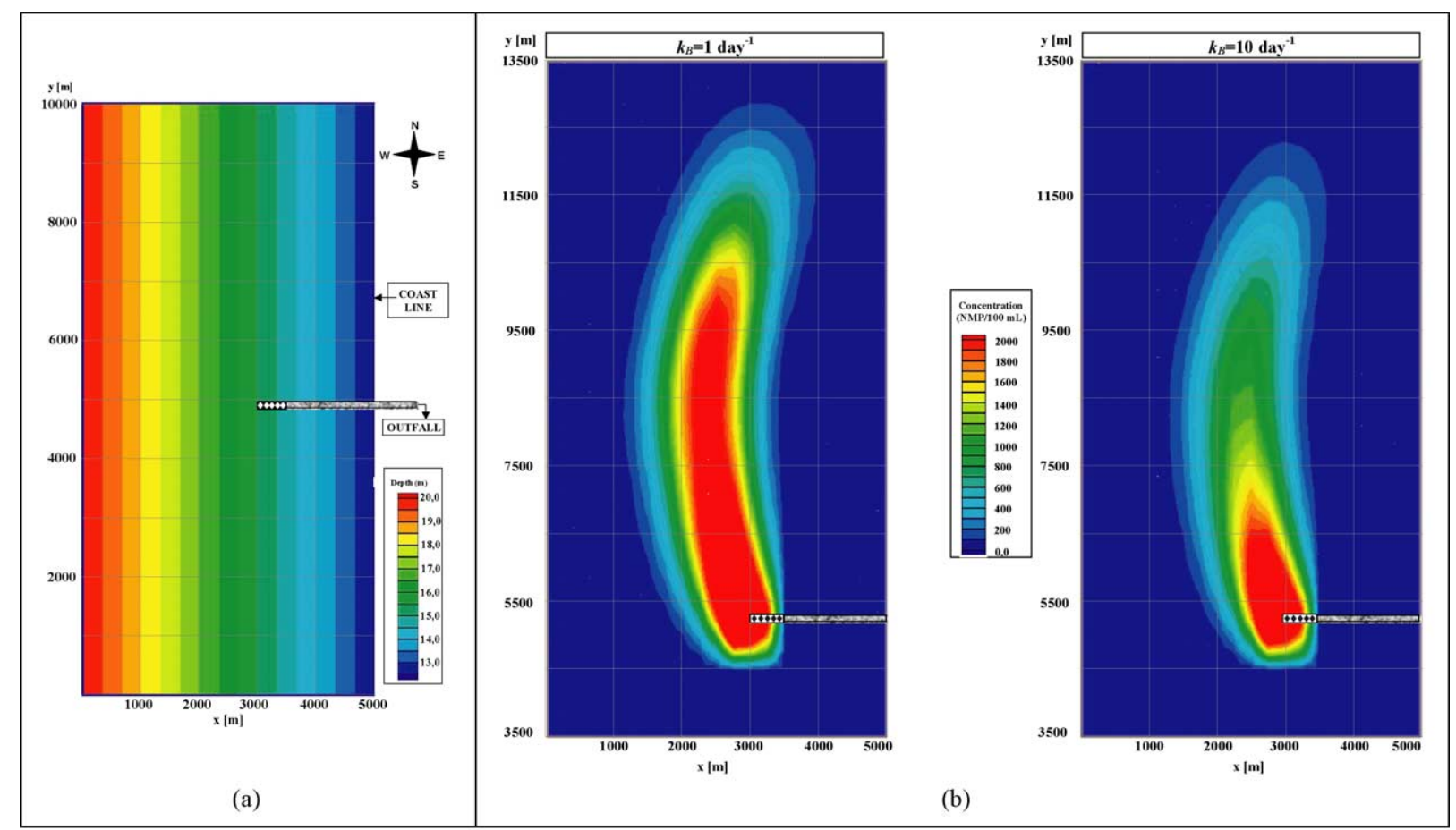

Fig. 18. POM-UMQ program application: (a) bottom topography; (b) submarine outfall plumes (plant), for different coliform bacteria decay coefficients, for a South-North alongshore current.

The possibility of simulating real hydrodynamic and water quality processes in coastal waters, shown in the previous presented results and other worked applications, reveals the enormous potentialities of the tool described for studying complex coastal water environmental engineering problems.

\subsection{GIS model results and data integration}

Sampling data and model results are first associated with the sampling station points, and then related to the mesh elements' nodes. Variable mapping thus implies a point-toarea transformation. This transformation must be twodirectional to allow the initial conditions to be used in a model simulation to be established. For example, by sampling data interpolation it is possible to define initial conditions for a variable within a grid or mesh, when it would be an area-topoint transformation. The present work employed a point-toarea transformation, without interpolations [16].

The methodology for integrating the geo-referenced hydrodynamics and water quality data, shown in Fig. 20, has

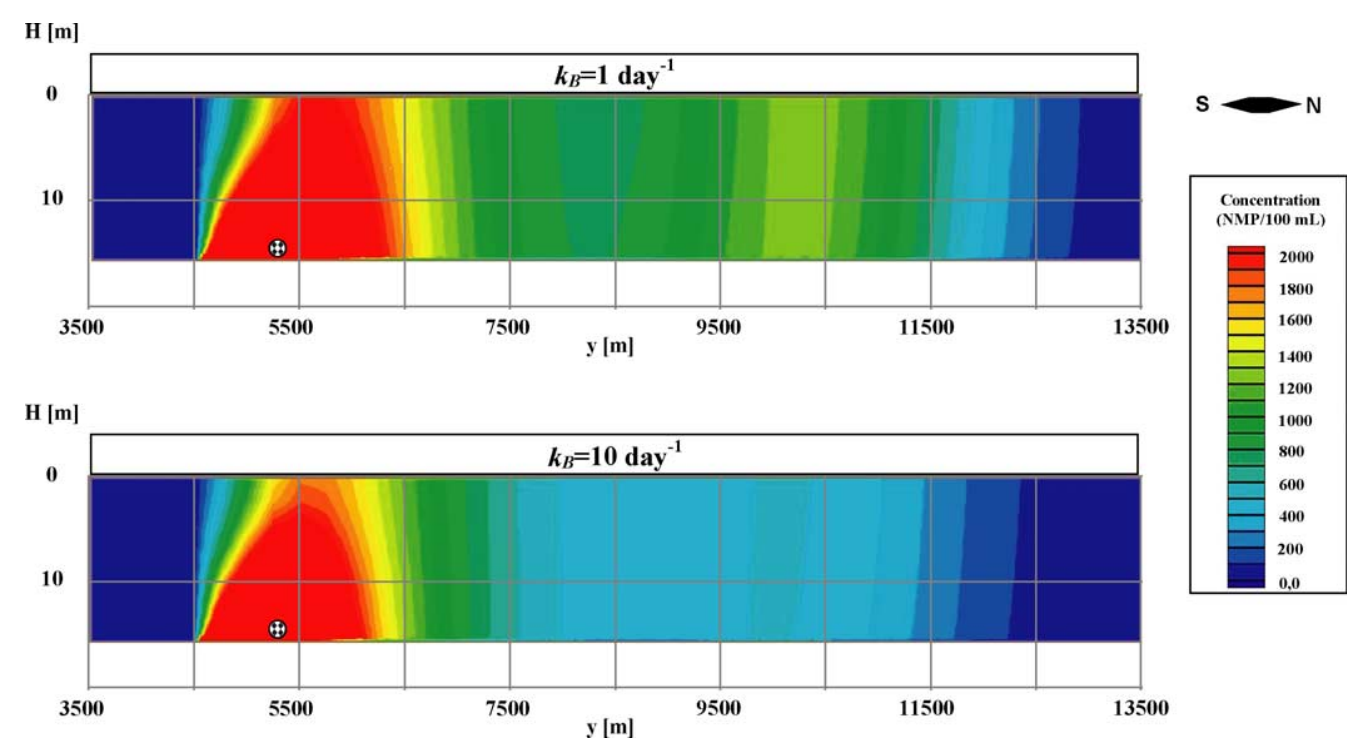

Fig. 19. POM-UMQ program application: submarine outfall plumes (vertical cuts), considering different coliform bacteria decay coefficients, for a South-North alongshore current. 


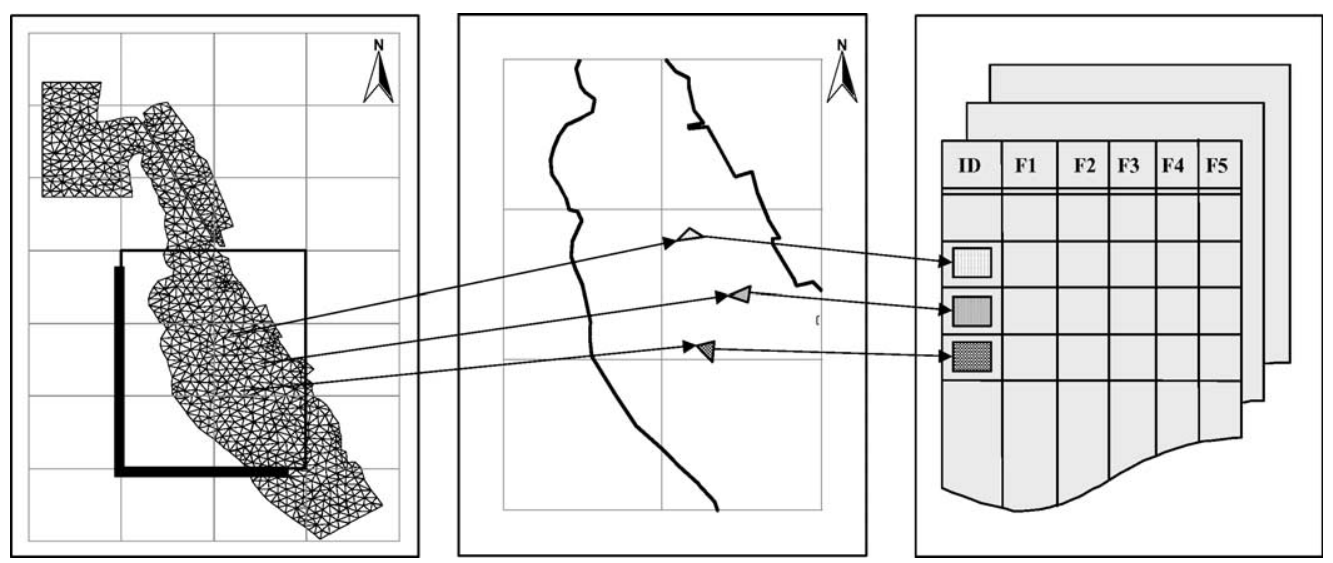

Fig. 20. Integration methodology of the geo-referenced hydrodynamics and water quality data.

the following main phases:

- the value of a variable within a finite element or a grid cell was considered constant (a single value, equal to the average of the element nodal values, was taken for each element); thus, the geometry file (models geometry) is transformed into graphical entities using a DXF file format;

- in the second phase, polygon entities are created with the GIS software, using the graphical entities in DXF format;

- next, the most important numerical models results are selected and organized, using a database tool;

- finally, the GIS themes are linked to the database results.

One of the GIS tools' potentialities is the capacity to organize and analyse different data sources in a common platform. It is thus possible to present and analyse sampling and monitoring data, etc., in the same numerical model system results. Fig. 21 gives the depth and velocity magnitude mapping at one instant of a hydrodynamic simulation, carried out using the RMA2 program, for river tavado estuary. Complementarily, a region was mapped for which the following conditions are simultaneously observed: velocity magnitude greater than $0.5 \mathrm{~m} / \mathrm{s}$ and depth greater than $1.0 \mathrm{~m}$.

Field data was mapped by means of a method based on the Thiessen polygons (also known as the Voronoi diagram) technique. An area of influence is defined for each sampling station according to the method described above, as illustrated in Fig. 22(a). This permits the mapping of any measured variable (assuming the simplification that the measured value is representative of the variable values within the polygon) and using the GIS potentialities to compare model data or newly derived data computations. For the example given (Fig. 22(b)), an estimation of the total mass of P1 (a general scalar variable) is computed using
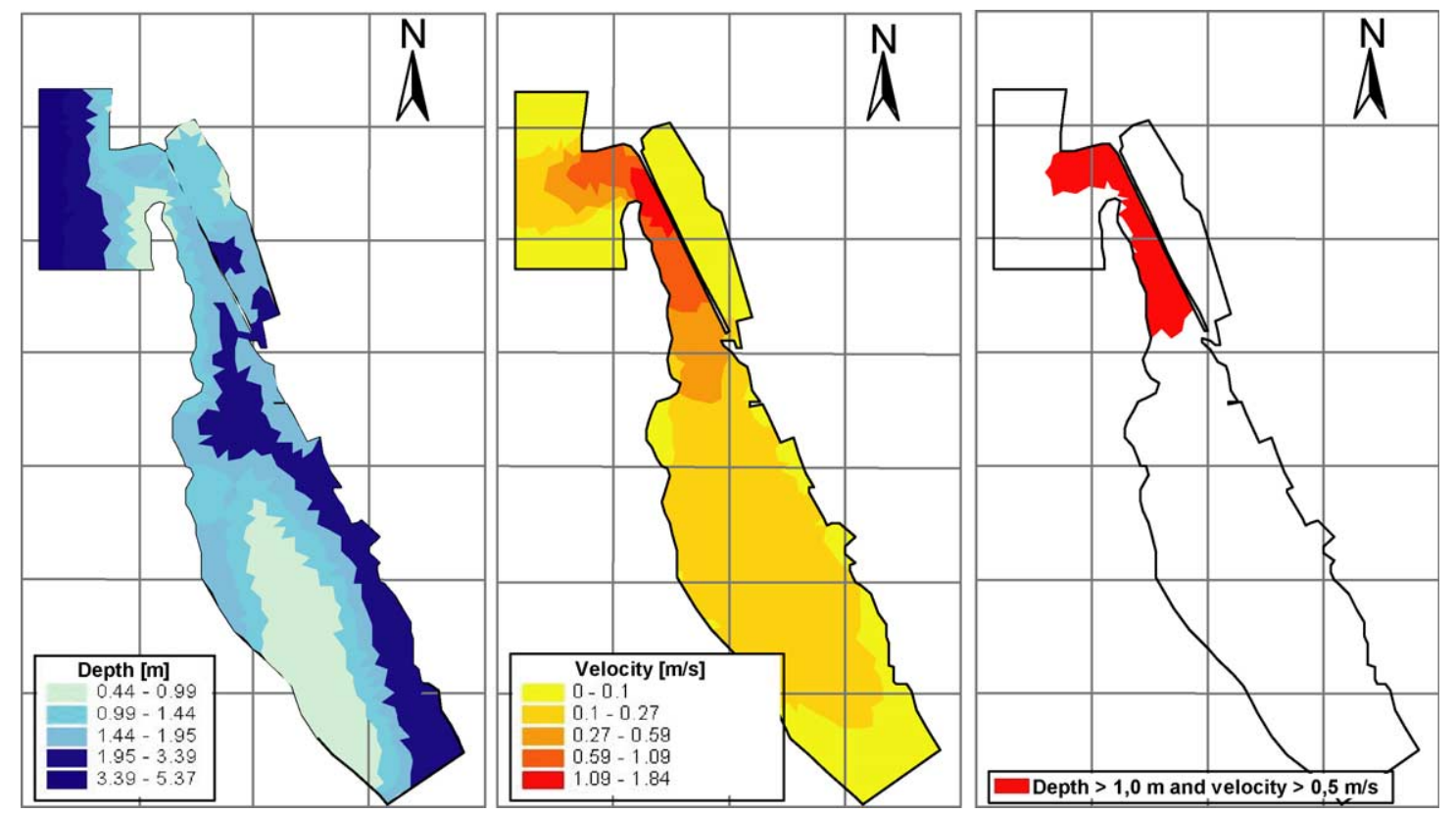

Fig. 21. Integration methodology for GIS numerical models' results. 

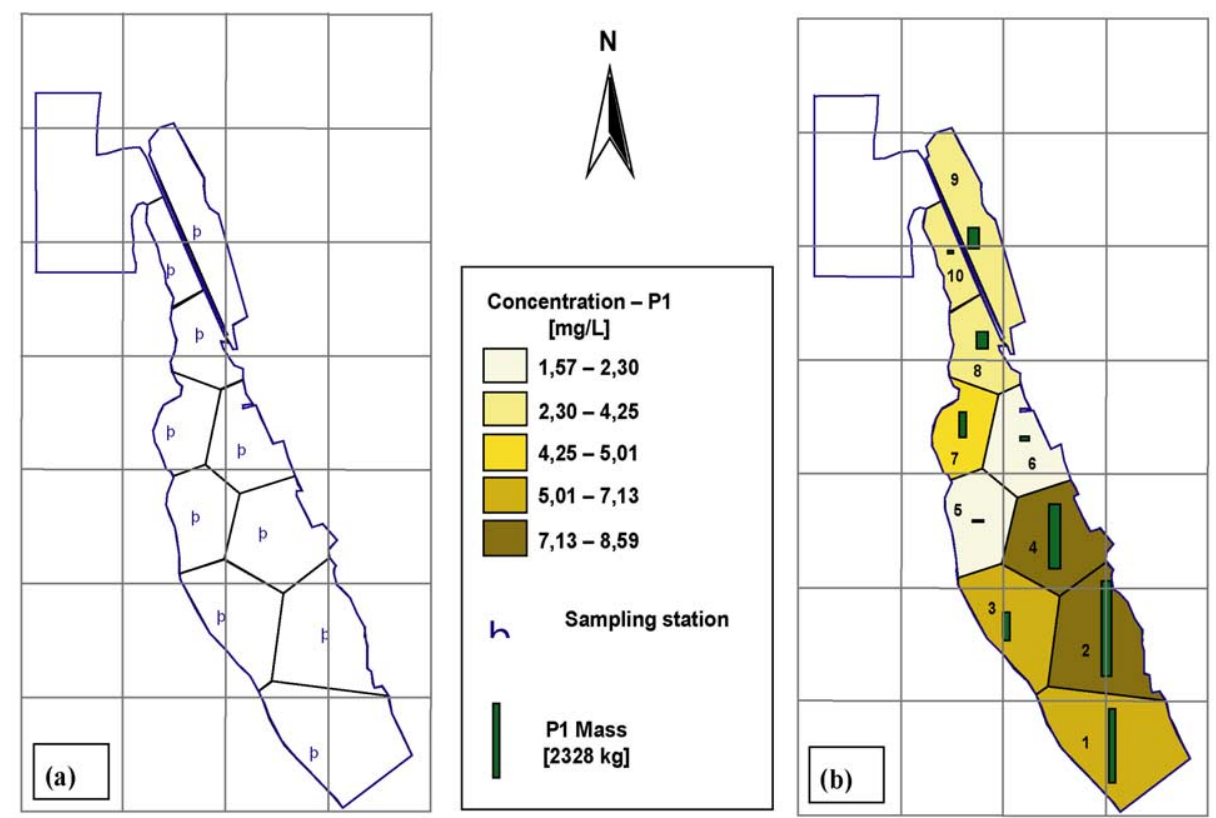

Fig. 22. Analysis of GIS field data and numerical models' results.

the concentrations measured at each station, as well as the area of each polygon and the water depth computed by the hydrodynamic model.

\section{Conclusions}

The success and quality of the results obtained with mathematical models depend equally on the techniques' efficiency and numerical methods used, and on the knowledge of the true capacities and limitations of the formulations introduced by modellers. The modular form adopted to create the hydroinformatic environment described in this work yields important savings when other programs or subroutines have to be included. Indeed, this methodology only requires the development of a specific tool to simulate a particular phenomenon. Furthermore, the definition of new model interfaces requires less work.

\section{References}

[1] Abbott MB. The sociotechnical dimension of hydroinformatics. In: Müller A, editor. Hydroinformatics'96, vol. 1. Rotterdam: A.A.Balkema; 1996. p. 3-18.

[2] Mellor G. Users guide for a three-dimensional, primitive equation, numerical ocean model. Princeton: Princeton University; 1998.

[3] Pinho JLS. Mathematical modelling application to hydrodynamics and water quality studies of coastal zones. PhD Thesis. University of Minho, Braga, Portugal (in Portuguese); 2001.
[4] BOSS SMS, Boss surface modeling system-user's manual. USA: Boss International, Inc. e Brigham Young University; 1996.

[5] ESRI, Using ArcView GIS. USA: Environmental Systems Research Institute, Inc; 1996.

[6] EDF (1991) Une présentation des équations de Saint-Venant, Electricité de France-Direction des Etudes et Recherches-Service Applications de l'Électricité et Environnement, Département Laboratoire National d'Hydraulique, Rapport EDF HE43/91.20, France

[7] WES-HL. Users Guide To RMA2 Version 4.3. US Army Corps of Engineers-Waterways Experiment Station Hydraulics Laboratory, Vicksburg, USA; 1996.

[8] Shewchuk JR. Triangle: Engineering a 2D Quality Mesh Generator and Delaunay Triangulator. Pittsburgh, USA: School of Computer Science, Carnegie Mellon University; 1997.

[9] JPL. A collection of global ocean tide models. Jet Propulsion Laboratory, Physical Oceanography Distributed Active Archive Center, Pasadena, CA; 1996. URL: http://podaac.jpl.nas.gov/

[10] Bern M, Eppstein D. Mesh generation and optimal triangulation. In: Du D-Z, Hwang FK, editors. Computing in Euclidean geometry, 2nd ed. Singapore: World Scientific; 1995.

[11] Ruppert J. A Delaunay refinement algorithm for quality 2-dimensional mesh generation. J Algorithms 1995;18(3):548-85.

[12] Otto L. Oceanography of the Ria de Arosa (N.W. Spain). Leiden University, NL; 1975.

[13] Gallego JG. Estudio de las condiciones oceanograficas en la Ria de Arosa, en Verano. Bol. Inst. Espanol de Oceanografia, 147; 1971. p. 39.

[14] Antunes do Carmo JS, Seabra-Santos FJ. On breaking waves and wave-current interaction in shallow water: a $2 \mathrm{DH}$ finite element model. Int J Numer Meth Fluids 1996;22:429-44.

[15] Thomann RV, Mueller JA. Principles of surface water quality modeling and control. USA: Harper Collins; 1987.

[16] Bonham-Carter GF. Geographic information systems for geoscientists-modelling with GIS. Computer Methods in the Geosciences, vol. 13. Canada: Pergamon Press; 1994. 